\title{
WestVirginiaUniversity
}

THE RESEARCH REPOSITORY @ WVU

Graduate Theses, Dissertations, and Problem Reports

2001

\section{Dynamic coloring of graphs}

\author{
Bruce Montgomery \\ West Virginia University
}

Follow this and additional works at: https://researchrepository.wvu.edu/etd

\section{Recommended Citation}

Montgomery, Bruce, "Dynamic coloring of graphs" (2001). Graduate Theses, Dissertations, and Problem Reports. 1397.

https://researchrepository.wvu.edu/etd/1397

This Dissertation is protected by copyright and/or related rights. It has been brought to you by the The Research Repository @ WVU with permission from the rights-holder(s). You are free to use this Dissertation in any way that is permitted by the copyright and related rights legislation that applies to your use. For other uses you must obtain permission from the rights-holder(s) directly, unless additional rights are indicated by a Creative Commons license in the record and/ or on the work itself. This Dissertation has been accepted for inclusion in WVU Graduate Theses, Dissertations, and Problem Reports collection by an authorized administrator of The Research Repository @ WVU.

For more information, please contact researchrepository@mail.wvu.edu. 


\title{
Dynamic Coloring of Graphs
}

\author{
Bruce Montgomery \\ Dissertation submitted to the \\ College of Arts and Sciences \\ at West Virginia University \\ in partial fulfillment of the requirements \\ for the degree of \\ Doctor of Philosophy \\ in \\ Mathematics \\ Hong-Jian Lai, Ph. D., Chair \\ Chong-Yun Chao, Ph. D. \\ Elaine Eschen, Ph. D. \\ John Goldwasser, Ph. D. \\ Dening Li, Ph. D. \\ Cun-Quan Zhang, Ph. D. \\ Department of Mathematics \\ Morgantown, West Virginia \\ 2001
}

Keywords: Graph Theory, Graph Colorings, Graph Structure, Dynamic Colorings

Copyright 2001 Bruce Montgomery 


\section{ABSTRACT \\ Dynamic Coloring of Graphs}

\section{Bruce Montgomery}

In this dissertation, we introduce and study the idea of a dynamic coloring of a graph, a coloring in which any multiple-degree vertex of the graph must be adjacent to at least two color classes.

As parts of the overall research, we study (for some interesting subjects of colorings) the corresponding subjects of dynamic colorings, we compare the chromatic number and dynamic chromatic number, and we study some problems unique to dynamic colorings. Also, we introduce and briefly study a generalization of dynamic coloring.

The interesting subjects of colorings we consider are the chromatic number of important graphs, upper bounds of the chromatic number, vertexcritical graphs, and stable graphs. For these first three subjects, we prove theorems for dynamic colorings that are similar to important theorems known for proper colorings, while we show no such theorems exist for stable graphs.

We make an extensive comparison of the two chromatic numbers that includes a description of graphs for which the two chromatic numbers are equal, that presents a class of graphs for which the per-graph differences in the two chromatic numbers is unbounded, that shows the difference is at most two for any $K_{1,3}$-free graph, and that studies the difference for regular graphs.

In our study of some unique problems of dynamic colorings, we characterize the graphs for which the dynamic chromatic number equals the number of vertices, we characterize the graphs for which the dynamic chromatic number 
equals one less than the number of vertices, we characterize the graphs for which the deletion of some vertex causes the dynamic chromatic number to decrease by more than one, and we obtain strong results describing graphs for which the removal of any vertex causes the dynamic chromatic number to increase.

Finally, we introduce and briefly study a generalization of dynamic coloring. 


\section{Acknowledgments}

I am grateful to Professor Hong-Jian Lai for his encouragement and advice. 


\section{Contents}

1 Introduction 1

1.1 General Idea . . . . . . . . . . . . . . . . . . . . . . 1

1.2 Definitions, Terminology, and Notation . . . . . . . . 2

2 Dynamic Chromatic Number of Particular Graphs 3

3 Upper Bounds $\quad 6$

$3.1 \chi_{d} \leq \Delta+3 \ldots \ldots \ldots \ldots \ldots$

$3.2 \chi_{d} \leq \alpha(G) \ldots \ldots \ldots \ldots \ldots \ldots$

4 Vertex-Critical Graphs $\quad 12$

4.1 Proper Colorings . . . . . . . . . . . . . . . . . . . . . . . . 12

4.2 Dynamic Colorings . . . . . . . . . . . . . . . . 13

5 Stable Graphs $\quad 16$

5.1 Proper Colorings . . . . . . . . . . . . . . . . 16

5.2 Dynamic Colorings . . . . . . . . . . . . . . . . . . . 19

6 Comparison of $\chi$ and $\chi_{d} \quad 22$

6.1 Normal Graphs . . . . . . . . . . . . . . . . . . . . 22

$6.2 \chi_{d}-\chi$ is Unbounded . . . . . . . . . . . . . . . . 24

$6.3 K_{1,3}$-Free Graphs . . . . . . . . . . . . . . 25 
6.4 Regular Graphs . . . . . . . . . . . . . . . . . . . . 28

7 Graphs with $\chi_{d}(G)=n$ or $n-1 \quad 30$

$8 \quad \chi_{d}(G-v)=\chi_{d}(G)-2 \quad 36$

9 Gainer Graphs $\quad 39$

10 A Generalization of Dynamic Coloring 43 


\section{List of Figures}

$\begin{array}{ll}\text { Figure 5.1 } & \text { Petersen Graph } \\ \text { Figure 5.2 } & G_{1} \\ \text { Figure 5.3 } & G_{2} \\ \text { Figure 5.4 } & G_{3} \\ \text { Figure 7.1 } & \chi_{d}(G)=n-1 \text { for } n=5 \\ \text { Figure } 9.1 & G_{1} \\ \text { Figure } 9.2 & G \text { for } l=4\end{array}$




\section{List of Symbols}

$\begin{array}{ll}c & \text { coloring } \\ c(v) & \text { color of vertex } v \\ d(v) & \text { degree of vertex } v \\ E(G) & \text { edge set of } G \\ G & \text { graph } \\ K_{n} & \text { complete graph on } n \text { vertices } \\ K_{i_{1}, \ldots, i_{l}} & \text { complete } l \text {-partite graph } \\ n & \text { number of vertices in a graph } \\ N(v) & \text { neighborhood of vertex } v \\ P_{k} & \text { path of length } k \\ v & \text { vertex } \\ V(G) & \text { vertex set of } G \\ & \\ \alpha(G) & \text { maximum length of cycles in } G \\ \Delta(G) & \text { maximum vertex degree } \\ \delta(G) & \text { minimum vertex degree } \\ \chi & \text { chromatic number } \\ \chi_{d} & \text { dynamic chromatic number } \\ \chi_{r} & \text { r-dynamic chromatic number } \\ G & \text { complement } \\ G-e & \text { deletion of an edge } \\ G-v & \text { deletion of a vertex } \\ & \end{array}$




\section{Chapter 1}

\section{Introduction}

\subsection{General Idea}

This dissertation proposes the idea of a dynamic coloring of a graph, which we now motivate and define.

A graph coloring assigns distinct colors to adjacent vertices. A coloring of the graph in which a typical vertex is adjacent to more than one color class represents a situation in which the typical individual has a greater variety in the types of relationships. Thus, the overall interactions would not be so limited but more dynamic.

Hence, a dynamic coloring is defined as a proper coloring for which any vertex of degree at least two is adjacent to more than one color class. As in the case of graph colorings, a chromatic number for dynamic colorings may similarly be defined.

Of course, any interesting problems of proper colorings may be reconsidered for dynamic colorings-the additional condition gives a fresh perspective. And the new condition creates significant problems that are unique for dynamic colorings. 


\subsection{Definitions, Terminology, and Notation}

In this section, the definitions, terminology, and notation used throughout are described. Terminology especially relevant to a particular section but not to previous sections will first be described in that section. With few exceptions, the definitions, terminology, and notation used here is consistent with that found in [2].

All graphs are finite and simple graphs. A graph with just one vertex is called trivial. Any graph is assumed to be connected, although the removal of edges or vertices may result in a graph that is not connected.

A graph is typically denoted by $G$ with vertex set $V=V(G)$ and edge set $E=E(G)$, with $n$ denoting $|V|$. The neighbor set $N(v)$ of a vertex $v$ is the set of vertices adjacent to $v$. The degree of $v$ is denoted by $d(v)$. The minimum vertex degree is denoted by $\delta=\delta(G)$, and the maximum vertex degree is denoted by $\Delta=\Delta(G)$. A $k$-coloring of $G$ is an assignment of $k$ colors $1,2, \ldots, k$ to the vertices of $G$. The $k$-coloring is proper if no two adjacent vertices are assigned the same color. The chromatic number $\chi=\chi(G)$ is the minimum $k$ for which $G$ has a proper $k$-coloring. A coloring of $G$ is typically a $\chi$-coloring of $G$.

A dynamic coloring is defined as a proper coloring in which any multipledegree vertex is adjacent to more than one color class. A dynamic coloring is thus a map $c$ from $V$ to the set of colors such that

(C1) If $u v \in E(G)$, then $c(u) \neq c(v)$, and

(C2) For each vertex $v \in V(G),|c(N(v))| \geq \min \{2, d(v)\}$

We call the first condition, which characterizes proper colorings, the adjacency condition, and we call the second condition the double-adjacency condition. The dynamic chromatic number $\chi_{d}=\chi_{d}(G)$ is the minimum $k$ for which $G$ has a dynamic $k$-coloring. The term dynamic coloring at times denotes a dynamic $\chi_{d}$-coloring, which should be clear from context. 


\section{Chapter 2}

\section{Dynamic Chromatic Number of Particular Graphs}

In this chapter, the dynamic chromatic number is obtained for complete graphs, trees, complete bipartite graphs, and cycles.

First note the simple fact that any graph has a dynamic coloring, since a coloring for which each vertex is colored differently satisfies the adjacency and double-adjacency conditions, and so minimizing over a nonempty set determines a dynamic coloring. Thus, $\chi_{d}(G) \leq n$ for any graph $G$.

Since any dynamic coloring is a proper coloring, $\chi_{d}(G) \geq \chi(G)$ for any graph $G$. Thus, $\chi_{d}\left(K_{n}\right)=n$.

Theorem 1 Let $G$ be a connected, nontrivial graph. Then $\chi_{d}\left(K_{2}\right)=2$ and $\chi_{d}(G) \geq 3$ otherwise.

Proof: If $n=2$, then $G=K_{2}$ and $\chi_{d}\left(K_{2}\right)=2$.

Otherwise, $n \geq 3$ and since $G$ is connected, then $G$ has a path of length two as a subgraph. Hence, some vertex $v$ on the path has two or more neighbors, and in a dynamic coloring at least two of them have different colors 
by the double-adjacency condition. Since those colors must be different from the color assigned to $v$, then $\chi_{d}(G) \geq 3$.

The path of $n$ vertices is denoted by $P_{n}$.

Theorem 2 For any tree $G$ other than $K_{1}$ or $K_{2}, \chi_{d}(G)=3$.

Proof: The proof is by induction on $n$.

For $n=1$ or $2, G=K_{n}$ and $\chi_{d}\left(K_{n}\right)=n$.

For $n=3, G=P_{3}$ and $\chi_{d}\left(P_{3}\right)=3$.

For a tree $G$ of $n \geq 4$ vertices, the tree $G-v$, where $d(v)=1$, satisfies $\chi_{d}(G-v)=3$ by the induction hypothesis. Given a dynamic three-coloring $c$ of $G-v$, color $v$ the third color distinct from $c(u)$ and $c(w)$, where $u$ is the vertex adjacent to $v$ and $w$ is a vertex other than $v$ adjacent to $u$. This yields a dynamic three-coloring of $G$, and so $\chi_{d}(G)=3$, since $\chi_{d}(G) \geq 3$ by Theorem 1.

The complete bipartite graph $K_{i, j}$ is the bipartite graph that has $V\left(K_{i, j}\right)$ partitioned as $X_{1} \cup X_{2}$ with $\left|X_{1}\right|=i,\left|X_{2}\right|=j$ and has each vertex in $X_{1}$ adjacent to each vertex in $X_{2}$.

Theorem 3 If $i, j \geq 2$, then $\chi_{d}\left(K_{i, j}\right)=4$.

Proof: Since $d(v) \geq 2$ for any vertex, then by the double-adjacency condition, at least two colors must be used to color $X_{1}$ and $X_{2}$ individually. Since by the adjacency condition, the vertices of $X_{1}$ and $X_{2}$ may share no common color, then at least $2+2=4$ colors are needed in any dynamic coloring of $K_{i, j}$. Clearly, a coloring which colors vertices of $X_{1}$ from $\{1,2\}$ and vertices of $X_{2}$ from $\{3,4\}$, and which uses all four colors, is a dynamic coloring.

The complete l-partite graph $K_{i_{1}, \ldots, i_{l}}$ is defined analogously for $l \geq 3$. Since $\chi\left(K_{i_{1}, \ldots, i_{l}}\right)=l$ and the proper coloring $c$ having $c\left(X_{j}\right)=j$ is clearly a dynamic coloring, then $\chi_{d}\left(K_{i_{1}, \ldots, i_{l}}\right)=l$ if $l \geq 3$.

An $n$-cycle is a cycle of $n$ vertices and is denoted by $C_{n}$. 


\section{Theorem 4}

$$
\chi_{d}\left(C_{n}\right)= \begin{cases}5 & \text { if } n=5 \\ 3 & \text { if } n=3 k, k \geq 1 \\ 4 & \text { otherwise }\end{cases}
$$

Proof: Let $v_{1} v_{2} \ldots v_{n} v_{1}$ denote an $n$-cycle of $C_{n}$. Note that any three consecutive vertices of the cycle must be colored differently in any dynamic coloring of $C_{n}$, since the first and third vertices are the only neighbors of the second vertex and must be colored differently (by the double-adjacency condition) and also differently from the second vertex.

Then $\chi_{d}\left(C_{3 k}\right)=3$ if $k \geq 1$, since $\chi_{d}(G) \geq 3$ and since the coloring $1,2,3, \ldots, 1,2,3,1$ of consecutive vertices is a dynamic three-coloring.

Since any three consecutive vertices must be colored differently, then $c\left(v_{1}\right), c\left(v_{2}\right)$, and $c\left(v_{3}\right)$ must be three different colors in a dynamic coloring $c$ of $C_{5}$. Then $c\left(v_{4}\right) \neq c\left(v_{1}\right), c\left(v_{2}\right), c\left(v_{3}\right)$ by the double-adjacency, doubleadjacency, and adjacency conditions, respectively. Finally, $c\left(v_{5}\right) \neq c\left(v_{1}\right)$, $c\left(v_{2}\right), c\left(v_{3}\right), c\left(v_{4}\right)$ by the adjacency, double-adjacency, double-adjacency, and adjacency conditions, respectively. Thus, all five vertices of $C_{5}$ must be colored differently, and so $\chi_{d}\left(C_{5}\right)=5$.

Let $k \geq 1$. Suppose $\chi_{d}\left(C_{3 k+1}\right)=3$. Since any three consecutive vertices must be colored differently, then, beginning with $1,2,3$, only the coloring $1,2,3,1,2,3, \ldots, 1,2,3, c\left(v_{3 k+1}\right), 1$ results. However, $c\left(v_{3 k+1}\right) \neq 1,2,3$ by the adjacency, double-adjacency, and adjacency conditions, respectively. Thus, $\chi_{d}\left(C_{3 k+1}\right) \geq 4$, and the coloring $1,2,3,1,2,3$, $\ldots, 1,2,3,4,1$ shows $\chi_{d}\left(C_{3 k+1}\right)=4$.

Let $k \geq 2$. If $\chi_{d}\left(C_{3 k+2}\right)=3$, then as argued before, only the coloring $1,2,3,1,2,3 \ldots 1,2,3,1, c\left(v_{3 k+2}\right), 1$ may be possible. However, $c\left(v_{3 k+2}\right) \neq$ 1,2,3 by the adjacency, double-adjacency, and double-adjacency conditions, respectively. Thus, $\chi_{d}\left(C_{3 k+2}\right) \geq 4$, and the coloring $1,2,3,1,2,3$, $\ldots, 4,2,1,4,3,1$ shows $\chi_{d}\left(C_{3 k+2}\right)=4$. 


\section{Chapter 3}

\section{Upper Bounds}

This chapter considers three of the most fundamental and important upper bounds known for $\chi(G)$. In each case, the upper bound is a function of a basic graph parameter. Thus, a natural question is whether an upper bound for $\chi_{d}(G)$ exists, in terms of the same parameter, that is otherwise similar to the upper bound for $\chi(G)$.

\section{$3.1 \chi_{d} \leq \Delta+3$}

The next theorem gives an upper bound for $\chi_{d}(G)$ that corresponds to the upper bound for $\chi(G)$ given by Brook's theorem $[1](\chi(G) \leq \Delta+1$, with equality holding only for complete graphs and odd cycles).

Theorem 5 For any graph $G, \chi_{d}(G) \leq \Delta+3$, and equality holds only for $G=C_{5}$.

Proof: Throughout the proof, $C(j)$ denotes a set of $j$ colors used by a typical coloring $c$ of $j$ colors $(C(j) \supset C(i)$ if $i<j)$.

Case 1: $\Delta=0$. Then $G$ is $K_{1}$, and so $\chi_{d}(G)=\Delta+1$.

Case 2: $\Delta=1$. Then $G$ is $K_{2}$, and so $\chi_{d}(G)=\Delta+1$. 
Case 3: $\Delta=2$. Then if $\mathrm{G}$ is acyclic, $G$ is a tree and so $\chi_{d}(G)=3=\Delta+1$. If $G$ contains a cycle, then $G$ is a cycle, and so $\chi_{d}(G) \leq 5=\Delta+3$, with equality only for $G=C_{5}$.

Case $4: \Delta=3$. We show that $\chi_{d}(G) \leq 5$.

The proof is by induction on $n$. The result is clearly true if $n \leq 5$, so suppose $n \geq 6$. In each case, a dynamic $k$-coloring $c$, where $k \leq 5$ is the dynamic chromatic number of the indicated graph, is extended to a dynamic $k^{\prime}$-coloring of the larger graph $G$, with $k \leq k^{\prime} \leq 5$.

Case $4 A: \delta=1$. Then if $d(v)=1$, extend a coloring $c$ of $G-v$ by choosing $c(v) \in C(5)-\{c(u), c(w)\}$, where $u$ is adjacent to $v$, and $w \neq v$ is adjacent to $u$.

Case $4 B: \delta=2$. Let $u$ and $v$ be neighbors and $d(v)=2$. Suppose first that $d(u)=2$. Suppose $v$ is adjacent to $v^{\prime} \neq u$, and $u$ is adjacent to $u^{\prime} \neq v$. Let $u^{\prime \prime} \neq u$ be some neighbor of $u^{\prime}$ and $v^{\prime \prime} \neq v$ be some neighbor of $v^{\prime}$. Extend a coloring $c$ of $G-\{u, v\}$ to a coloring of $G$ by choosing $c(u) \in C(5)-\left\{c\left(v^{\prime}\right), c\left(u^{\prime}\right), c\left(u^{\prime \prime}\right)\right\}$ and $c(v) \in C(5)-\left\{c(u), c\left(u^{\prime}\right), c\left(v^{\prime}\right), c\left(v^{\prime \prime}\right)\right\}$.

Suppose next that $d(u)=3$ and $u$ is adjacent to $u_{1}^{\prime}, u_{2}^{\prime}$, and $v$. If $d\left(u_{1}^{\prime}\right)=d\left(u_{2}^{\prime}\right)=3$, extend a coloring of $G-\{u, v\}$ by choosing $c(u) \in$ $C(5)-\left\{c\left(u_{1}^{\prime}\right), c\left(u_{2}^{\prime}\right), c\left(v^{\prime}\right)\right\}$ and $c(v) \in C(5)-\left\{c\left(u_{1}^{\prime}\right), c(u), c\left(v^{\prime}\right), c\left(v^{\prime \prime}\right)\right\}$. If $d\left(u_{1}^{\prime}\right)=2$ or $d\left(u_{2}^{\prime}\right)=2$, then extend a coloring $c$ of $G-\left\{v^{\prime}, v\right\}$ by choosing $c(v) \in C(5)-\left\{c(u), c\left(v^{\prime}\right), c\left(v^{\prime \prime}\right)\right\}$ and $c\left(v^{\prime}\right) \in C(5)-\left\{c(u), c(v), c\left(v^{\prime \prime}\right), c\left(v^{\prime \prime \prime}\right)\right\}$, where $v^{\prime \prime \prime} \neq v^{\prime}$ is adjacent to $v^{\prime \prime}$. This completes the $\delta(G)=2$ case.

Case $4 C: \delta(G)=\Delta(G)=3$. Then extend a coloring $c$ of $G-v$ for any $v \in V$ by choosing $c(v) \in C(5)-\{c(u), c(w), c(x)\}$, where $v$ is adjacent to $u, w$, and $x$. This completes the $\Delta=3$ case.

Case 5: $\Delta \geq 4$. We show $\chi_{d}(G) \leq \Delta+2$.

Let $V(G)=\left\{v_{1}, \ldots, v_{n}\right\}$, and let $G_{i}=G\left(\left\{v_{1}, \ldots, v_{i}\right\}\right)$. Note that any $G_{i}$ for $i \neq 1$ may not be connected.

We now show by induction on $\Delta \leq i \leq n-1$ that 
(1) $G_{i+1}$ has a $(\Delta+2)$-coloring if $\Delta+2-d_{G_{i+1}}\left(v_{i+1}\right) \geq 2$, in which $v_{i+1}$ can be colored with any of two or more colors if $\Delta+2-d_{G_{i+1}}\left(v_{i+1}\right) \geq 3$.

We denote as follows these various neighbor sets of $v_{i+1}$ in $G_{i+1}$ : those of degree at least two in $G_{i}$ as $X=\left\{x_{1}, \ldots, x_{k_{1}}\right\}$, those of degree one in $G_{i}$ as $Y=\left\{y_{1}, \ldots, y_{k_{2}}\right\}$, and those of degree zero in $G_{i}$ as $Z=\left\{z_{1}, \ldots, z_{k_{3}}\right\}$. Let $Y^{\prime}=\left\{y_{1}^{\prime}, \ldots, y_{k_{2}}^{\prime}\right\}$, where $y_{j}^{\prime}$ is the neighbor of $y_{j}$ in $G_{i}$.

We shall denote $\Delta+2-d_{G_{i+1}}\left(v_{i+1}\right)$ by $l$. Note that $l \leq \mid C(\Delta+2)-$ $(c(X) \cup c(Y) \cup c(Z)) \mid$.

Case 5A: $i=\Delta$. Then (1) holds, since if $c\left(v_{i}\right)=\sigma_{i}$ for $1 \leq i \leq \Delta$, then $c\left(v_{\Delta+1}\right)=\sigma_{\Delta+1}$ or $c\left(v_{\Delta+1}\right)=\sigma_{\Delta+2}$ each yield a dynamic coloring of $G_{\Delta+1}$.

Case 5B: $i \geq \Delta+1$. Let the current coloring of $G_{i}$ be denoted by $c^{\prime}$. Let $S=\left\{\sigma_{1}, \ldots, \sigma_{t}\right\}$, where $\sigma_{1}, \ldots, \sigma_{t}$ are the distinct colors in $c^{\prime}(Y)$ and $\left\{\sigma_{t+1}, \ldots, \sigma_{m}\right\}=C(\Delta+2)-c^{\prime}(X) \cup c^{\prime}(Z) \cup c^{\prime}\left(Y^{\prime}\right)$.

We obtain a new coloring $c$ of $G_{i}$ by recoloring $y_{1}, \ldots, y_{k_{2}}$ (for any other vertex of $G_{i}, c$ is identical to $\left.c^{\prime}\right)$. Let $c\left(y_{j}\right)=\sigma_{i_{j}}$ such that $\sigma_{i_{j}} \neq c^{\prime}\left(y_{j}^{\prime}\right)$ and the coloring of $y_{j}$ with $\sigma_{i_{j}}$ ensures that $y_{j}^{\prime}$ satisfies the double-adjacency condition.

Case 5B1: $\left|c^{\prime}\left(Y^{\prime}\right)\right| \geq 3$. Then at most one color $\beta^{\prime}$ from $c\left(Y^{\prime}\right)$ is not in $c(Y)$, since $\beta \in c\left(Y^{\prime}\right)$ and $\beta \notin c(Y)$ implies, for each $j, 1 \leq j \leq k_{2}$, $c\left(y_{j}^{\prime}\right)=\beta$ or $c\left(y_{j}^{\prime \prime}\right)=\beta$ for every $y_{j}^{\prime \prime} \neq y_{j}$ adjacent to $y_{j}^{\prime}$ in $G_{i}$. This could occur simultaneously for two colors $\beta$ but not for three or more.

Since $l \geq 2$, then there exists at least one choice to color $v_{i+1}$ from $C(\Delta+2)-\left(c(X) \cup c(Y) \cup c(Z) \cup\left\{\beta^{\prime}\right\}\right)$ if $l=2$ and at least two choices if $l \geq 3$. Each yields a dynamic coloring since, in particular, $\left|c\left(N_{G_{i+1}}\left(v_{i+1}\right)\right)\right| \geq$ $|c(Y)| \geq\left|c^{\prime}\left(Y^{\prime}\right)\right| \geq 3$ and $v_{i+1}$ colored differently from any $y_{j}^{\prime}$ ensures that each $y_{j}$ satisfies the double-adjacency condition.

Case 5B2: $\left|c^{\prime}\left(Y^{\prime}\right)\right|=2$. Then at most one color $\beta_{1} \in c^{\prime}\left(Y^{\prime}\right)$ is not in $c(Y)$, or neither of the colors $\beta_{1}$ and $\beta_{2}$ in $c^{\prime}\left(Y^{\prime}\right)$ is in $c(Y)$ (which occurs when, for each $j, 1 \leq j \leq k_{2}, y_{j}^{\prime}$ is adjacent in $G_{i}$ to only $y_{j}$ and $y_{j}^{\prime \prime}$, and either 
$c^{\prime}\left(y_{j}^{\prime}\right)=\beta_{1}$ and $c^{\prime}\left(y_{j}^{\prime \prime}\right)=\beta_{2}$, or $c^{\prime}\left(y_{j}^{\prime}\right)=\beta_{2}$ and $\left.c^{\prime}\left(y_{j}^{\prime \prime}\right)=\beta_{1}\right)$.

First consider $k_{1}=k_{3}=0$. Among the four or more colors in $C(\Delta+2)-$ $\left\{\beta_{1}, \beta_{2}\right\}$, we use two to color the vertices in $Y$, so that at least two choices remain to color $v_{i+1}$.

If $k_{1}>0$ or $k_{3}>0$, then among the $l+k_{2}$ or more colors in $C(\Delta+2)-$ $(c(X) \cup c(Z))$, use one not in $\left\{\beta_{1}, \beta_{2}\right\}$ to color the vertices in $Y$, so that at least two choices remain to color $v_{i+1}$ if $l \geq 3$ and at least one choice if $l=2$.

Case 5B3: $\left|c^{\prime}\left(Y^{\prime}\right)\right|=1$. If $|c(X) \cup c(Z)| \geq 1$, then, as in the last case, use a color not in $c^{\prime}\left(Y^{\prime}\right)$ to color the vertices in $Y$, so that at least two choices remain to color $v_{i+1}$.

Case 5B4: $\left|c^{\prime}\left(Y^{\prime}\right)\right|=0$. Thus $k_{2}=0$. Suppose $k_{3}>0$.

If $|c(X) \cup c(Z)| \geq 2$, then $v_{i+1}$ can be colored with any of the $l \geq 2$ or more colors in $C(\Delta+2)-(c(X) \cup c(Z))$ to obtain a $(\Delta+2)$-coloring of $G_{i+1}$.

If $|c(X) \cup c(Z)|=1$, then of the five colors in $C(\Delta+2)-(c(X) \cup c(Z))$, recolor $z_{1}$ with one and color $v_{i+1}$ with any of the 4 other colors to obtain a $(\Delta+2)$-coloring of $G_{i+1}$.

Finally, suppose $k_{3}=0$.

If $|c(X)| \geq 2, v_{i+1}$ can be colored with any of the $l \geq 2$ or more colors in $C(\Delta+2)-c(X)$ to obtain a $(\Delta+2)$-coloring of $G_{i+1}$.

If $|c(X)|=1$, let $\left\{x_{1}, \ldots, x_{k_{1}}\right\}=\left\{v_{i_{1}}, \ldots, v_{i_{k_{1}}}\right\}$, with $i_{1}<\ldots<i_{k_{1}}=i$. Since $v_{i}$ is adjacent to $v_{i+1}$, then $\Delta+2-d_{G_{i}}\left(v_{i}\right) \geq 3$, and so by induction we can recolor $v_{i}$ to obtain the $|c(X)| \geq 2$ case. This completes the proof of the $\Delta(G) \geq 4$ case, and thus completes the proof of $\chi_{d}(G) \leq \Delta(G)+3$ for any graph $G$.

The bound is achieved with equality only for $G=C_{5}$, since we have shown that if $\Delta(G) \neq 2, \chi_{d}(G) \leq \Delta+2$, and if $\Delta(G)=2, \chi_{d}(G)=5$ only for $G=C_{5}$. 


\section{$3.2 \chi_{d} \leq \alpha(G)$}

A well-known upper bound for $\chi(G)$ in terms of the length $\alpha(G)$ of a longest cycle in $G$ is (Corollary 8.8 of [3], p. 226): $\chi(G) \leq \alpha(G)$. This bound holds with equality for $K_{n}$, since $\chi\left(K_{n}\right)=n=\alpha\left(K_{n}\right)$ for any $n \geq 3$.

Theorem 6 If $G$ has a cycle, then $\chi_{d}(G) \leq \alpha(G)$.

Proof: The proof is by induction on $n$.

The theorem is clearly true for $n \leq 3$, so suppose $n \geq 4$ and $G$ has a cycle. Let $v$ be a terminal vertex of a longest path $P$ in $G$, say $P=v v_{1} v_{2} \ldots v_{s}$, where $s \geq \alpha(G)-1$. Then $G-v$ is also connected. If $G-v$ has no cycles for every such choice of $v$, then $G$ is a cycle, and so $\chi_{d}(G) \leq \alpha(G)$. So, suppose $G-v$ has a cycle. Thus, $\chi_{d}(G-v) \leq \alpha(G-v) \leq \alpha(G)$.

Note that $v$ may be adjacent only to vertices on $P$ (since otherwise $G$ has a longer path than $P$ ) and in particular only to vertices in $A=\left\{v_{1}, \ldots, v_{\alpha(G)-1}\right\}$ (since otherwise $G$ has a cycle of length greater than $\alpha(G)$ ). By the same argument, $N_{G}(W) \subset A$ for $W=N_{G}\left(v_{1}\right)-\left\{v, v_{2}, \ldots, v_{\alpha(G)}\right\}$.

Suppose $c_{1}$ is a dynamic coloring of $G-v$ of at most $\alpha(G)$ colors from $C(\alpha(G))$. Let $c$ be a coloring of $G$ defined by $c(u)=c_{1}(u)$ if $u \notin W \bigcup\left\{v, v_{1}\right\}$; $c(w)=c(v)$ is chosen from $C(\alpha(G))-c\left(\left\{v_{2}, \ldots, v_{\alpha(G)-1}\right\}\right)$ for any $w \in W$, taken to be $c\left(v_{\alpha(G)}\right)$ if one such choice; and $c\left(v_{1}\right)$ is chosen from $C(\alpha(G))-$ $c\left(\left\{v, v_{2}, \ldots, v_{\alpha(G)}\right\}\right)$ (nonempty because $c(v)=c\left(v_{i}\right)$ for some $i, 2 \leq i \leq$ $\alpha(G))$. Thus, at most $\alpha(G)$ colors are necessary to define $c$. Note that $c(w), c(v) \notin c(A)$ for any $w \in W$.

We now show that $c$ is a dynamic coloring. Note that for any $w \in W$, $A$ contains $N_{G}(v) \cup N_{G}(w)$ and $A$ contains a neighbor of $x$ for each vertex $x \in N_{G}(v) \cup N_{G}(w)$. Thus, $v$ and $w$ satisfy the adjacency condition, since $c(w)=c(v) \notin c(A) ; v$ and $w$ satisfy the double-adjacency condition, since $v$ and $w$ are adjacent to $v_{1}$ while $c\left(v_{1}\right) \notin c\left(A-\left\{v_{1}\right\}\right)$ and $A \supset N_{G}(v), N_{G}(w)$. 
Also, any neighbor $x$ of $v$ or $w$ satisfies the double-adjacency condition, since $c(w)=c(v) \notin c(A)$ and $A$ contains a neighbor of $x$.

Note that $B=\left\{v, v_{2}, \ldots, v_{\alpha(G)}\right\} \cup W$ contains $N_{G}\left(v_{1}\right)$. Thus, $v_{1}$ satisfies the adjacency condition, since $c\left(v_{1}\right) \notin c(B)$; $v_{1}$ satisfies the double-adjacency condition, since $v_{1}$ is adjacent to $v$ and $v_{2}$ but $c(v) \neq c\left(v_{2}\right)$. Note that $B$ contains, for each vertex $x \neq v, w$ in $N_{G}\left(v_{1}\right)$, a neighbor of $x$. Thus, any neighbor $x \neq v, w$ of $v_{1}$ satisfies the double-adjacency condition, since $c\left(v_{1}\right) \notin c(B)$ and $B$ contains a neighbor of $x$.

Finally, since $c_{1}$ is a dynamic coloring of $G-v$, the conditions of a dynamic coloring for vertices other than $v, v_{1}, w$, and their neighbors hold also for $c$.

The bound holds with equality for such graphs as $C_{4}, C_{5}$, and $K_{n}$ for all $n \geq 3$.

A well-known upper bound for $\chi(G)$ in terms of the length $l(G)$ of a longest path in $G$ is given in $[3]: \chi(G) \leq l(G)+1$. The bound holds with equality for $K_{n}$ for any $n \geq 2$.

As a corollary of the previous theorem, we obtain an identical upper bound for $\chi_{d}(G)$.

Corollary 1 For any graph $G, \chi_{d}(G) \leq l(G)+1$.

Proof: The result is clearly true for $n=1$ and $n=2$. So suppose $n \geq 3$. If $G$ is acyclic, then $G$ is a tree, and so $\chi_{d}(G)=3 \leq l(G)+1$, since a tree has a path of length 2 if $n \geq 3$. If $G$ contains a cycle, then since any cycle contains a path with length one less than the cycle length, $\chi_{d}(G) \leq \alpha(G) \leq l(G)+1$.

The bound holds with equality for such graphs as $C_{4}, C_{5}$, and $K_{n}$ for all $n \geq 2$. 


\section{Chapter 4}

\section{Vertex-Critical Graphs}

\subsection{Proper Colorings}

A vertex-critical graph for proper colorings is defined to be a graph $G$ such that $\chi(G-v)<\chi(G)$ for any vertex $v$ of $G$. Graphs having much symmetry are expected to be among the best candidates. So, it is perhaps not surprising to find that all complete graphs and all odd cycles are vertex-critical graphs, since $\chi\left(K_{n}-v\right)=n-1=\chi\left(K_{n}\right)-1$ for any $n \geq 1$ and $\chi\left(C_{n}-v\right)=2=$ $\chi\left(C_{n}\right)-1$ for any odd $n \geq 3$.

Any graph $G=K_{2 k-1}-E\left(C_{2 k-1}\right)$ for $\chi(G)=k \geq 3$ is also a vertexcritical graph, since for $k \geq 4$ the consecutive vertices of $C_{2 k-1}$ may be colored as $1,1,2,2, \ldots, k-1, k-1, k$ in an optimal coloring of $G$, and the consecutive vertices of $P_{2 k-2}$ of $G-v=K_{2 k-2}-E\left(P_{2 k-2}\right)$ may be colored as $1,1,2,2, \ldots, k-1, k-1$. (For $k=3, G=K_{5}-E\left(C_{5}\right)=C_{5}$ is an odd cycle.)

It is now shown that complete graphs and odd cycles are sufficient to characterize the vertex-critical graphs if $\chi(G)=2,3$, or $n$.

Theorem 7 Let $G$ be a graph of $n$ vertices. Then $G$ is a vertex-critical graph with $\chi(G)=2$ if and only if $G=K_{2}, G$ is a vertex-critical graph with 
$\chi(G)=3$ if and only if $G$ is an odd cycle, and $G$ is a vertex-critical graph with $\chi(G)=n$ if and only if $G=K_{n}$ and $n \geq 1$.

Proof: Let $G$ be a vertex-critical graph with $\chi(G)=2$. Since $\chi(G-v)=1$, then $G-v$ consists of one or more isolated vertices, so that $G=K_{1, n-1}$. But $\chi\left(K_{1, j}-v\right)=1$ only for $v$ the central vertex, so that $G=K_{1,1}=K_{2}$. Of course, $\chi\left(K_{2}\right)=2$.

Let $G$ be a vertex-critical graph with $\chi(G)=3$. Thus, $G$ has an odd cycle $C$. If there exists a vertex $v$ not on $C$, then since $G-v$ contains $C$, an odd cycle, then $\chi(G-v) \neq 2$, and $G$ would not be vertex critical. Thus, there is no vertex not on $C$, i. e., $G=C_{n}$ and $n$ is odd. The converse was previously noted.

Let $G$ be a vertex-critical graph with $\chi(G)=n$. Since $\chi(G)=n$, any two vertices are adjacent, since otherwise a pair of nonadjacent vertices could be colored the same color different from each of the $n-2$ colors used to uniquely color each of the remaining vertices. Thus, $G=K_{n}$. The converse was previously noted.

\subsection{Dynamic Colorings}

A graph $G$ is defined to be a vertex-critical graph for dynamic colorings if $\chi_{d}(G-v)<\chi_{d}(G)$ for any vertex $v$ of $G$.

It is also true for dynamic colorings that all complete graphs are vertex critical. However, as is readily checked from $\chi_{d}\left(C_{n}\right), C_{n}$ is a vertex-critical graph if and only if $n$ is not a multiple of three, so that a significantly higher fraction (two-thirds) of these graphs are vertex critical in the dynamic case as compared to the proper case (one-half).

Any graph $G=K_{2 k-1}-E\left(C_{2 k-1}\right)$ for $k=\chi_{d}(G) \geq 4$ is a vertex-critical graph for dynamic colorings, since the proper coloring specified for $G$ and $G-v$ is in each case also a dynamic coloring. 
The Petersen graph $\left(\chi_{d}(G)=4, \chi(G)=3\right)$ is vertex critical for dynamic colorings but not for proper colorings.

An example of a class of graphs vertex critical for dynamic colorings but not for proper colorings is $G=S K_{k}$ for $k=\chi_{d} \geq 4$. $G$ is a bipartite graph with $V(G)$ partitioned as $V(G)=X \cup Y$. Each of the $\left(\begin{array}{l}k \\ 2\end{array}\right)$ vertices in $Y$ is adjacent to a unique pair of the $k$ vertices in $X=\left\{x_{1}, \ldots, x_{k}\right\}$. Thus, $\chi_{d}(G) \geq k$, since any two vertices in $X$ must be colored differently, being the only two vertices adjacent to some vertex in $Y$. Let $c\left(x_{i}\right)=i$ and, if $y$ in $Y$ is adjacent to $x_{i}$ and $x_{j}$ in $X$, let $c(y) \in\{1, \ldots, k\}$ such that $c(y) \neq i, j$. Thus, no $x_{i}$ is adjacent to only vertices of the same color $l$, since some $y$ in $Y$ is adjacent to $x_{i}$ and $x_{l}$ and hence has $c(y) \neq i, l$. Hence, $c$ is clearly a dynamic coloring of $G$, and so $\chi_{d}\left(S K_{k}\right)=k$. Let $x_{m} \in X$. Consider a coloring $c$ that is a 1-1 map of $X-\left\{x_{m}\right\}$ onto $\{1, \ldots, k-1\}$ and for which $c(y) \in\{1, \ldots, k-1\}$ and $c(y) \neq c\left(x_{i}\right), c\left(x_{j}\right)$ if $y$ is adjacent to $x_{i}$ and $x_{j}$, $c(y) \neq c\left(x_{i}\right)$ if $y$ is adjacent only to $x_{i}$. Then $c$ is a dynamic coloring of $G-x$. Thus, $\chi_{d}\left(G-x_{m}\right)=k-1$, since any two of the $k-1$ vertices of $X-x_{m}$ must be colored differently, being the only two vertices adjacent to some vertex in $Y$. Also, $\chi_{d}(G-z)=k-1$, where $z$ in $Y$ is adjacent to $x_{i}$ and $x_{j}$ in $X$ : first, a coloring $c$ is a dynamic coloring of $G-z$ if (except for $c\left(x_{i}\right)=c\left(x_{j}\right)$ ) $c$ is a 1-1 map of $X$ onto $\{1,2, \ldots, k-1\}$ and has $c(y) \neq c\left(x_{l}\right), c\left(x_{m}\right)$ if $y$ in $Y-\{z\}$ is adjacent to $x_{l}$ and $x_{m}$; second, at least $k-1$ colors are required, since any two vertices of $X-x_{i}$ must be colored differently, being the only two vertices adjacent to some vertex in $Y-\{z\}$.

As was true for proper colorings, it is also possible to characterize the vertex-critical graphs for dynamic colorings if $\chi_{d}(G)=2,3$, or $n$.

Theorem 8 Let $G$ be a graph of $n$ vertices. Then $G$ is a vertex-critical graph with $\chi_{d}(G)=2$ if and only if $G=K_{2}, G$ is a vertex-critical graph with $\chi_{d}(G)=3$ if and only if $G=K_{3}$ or $P_{3}$, and $G$ is a vertex-critical graph with 
$\chi_{d}(G)=n$ if and only if $G=P_{3}, C_{4}, C_{5}$, or $K_{n}$.

Proof: Since $\chi_{d}(G)=2$ if and only if $G=K_{2}$ and since $\chi_{d}\left(K_{2}-v\right)=1$ for each vertex $v$, then the first part is shown.

Clearly, for $n \leq 3, G=K_{3}$ and $G=P_{3}$ are the only vertex-critical graphs with $\chi_{d}(G)=3$. Let $n \geq 4$ and let $G$ be a vertex-critical graph with $\chi_{d}(G)=3$. Since $\chi_{d}(G-v)=2$ for any vertex $v$, then any component of $G-v$ is $K_{1}$ or $K_{2}$. However, if some component is $K_{1}=\{u\}$, then $d(u)=1$ and thus $\chi_{d}(G-u) \geq 3$, since $G-u$ is a connected graph of at least three vertices. So, $G$ has minimum degree of at least two. Thus, each component of $G-v$ is $K_{2}$ and $v$ is adjacent to each vertex, so that $G$ consists of triangles with $v$ a vertex of each triangle and otherwise no two triangles sharing a vertex. However, any vertex $u$ other than $v$ is not a cut vertex of $G$, and so $\chi_{d}(G-u) \geq 3$ and hence $G$ is not vertex-critical.

Finally, by Theorem 17, $\chi_{d}(G)=n$ if and only if $G=P_{3}, C_{4}, C_{5}$, or $K_{n}$, and each has been shown to be vertex critical for dynamic colorings.

The vertex-critical graphs for dynamic colorings with $\chi_{d}(G)=4$ may be quite difficult to characterize since already they have been shown to include such a wide variety of graphs as $C_{n}$ ( $n$ not equal to 5 and $n$ not a multiple of 3$), K_{4}, S K_{4}, K_{9}-E\left(C_{9}\right)$, and the Petersen graph. However, one property that can be proved is that $\delta(G) \geq 2$. ( Suppose $\delta(G)=1$ and let $d(v)=1$. Since $\chi_{d}(G-v)=3$, color $v$ differently from its adjacent vertex $u$ and differently from some vertex $w \neq v$ adjacent to $u$ to extend a dynamic 3coloring of $G-v$ and obtain a dynamic 3-coloring of $G$, a contradiction.) 


\section{Chapter 5}

\section{Stable Graphs}

A graph $G$ is said to be stable for proper colorings if $\chi(G-v)=\chi(G)$ for any $v$, and is said to be stable for dynamic colorings if $\chi_{d}(G-v)=\chi_{d}(G)$ for any $v$. Thus, in either case, a stable graph is the antithesis of a vertex-critical graph.

\subsection{Proper Colorings}

We first consider stable graphs for proper colorings. Some examples of stable graphs when $\chi(G)=2$ are the graphs $C_{n}$ if and only if $n$ is even, and any tree if and only if $n \geq 4$ and other than $K_{1, n-1}$. (Since any tree of at least two vertices has chromatic number two, then a tree $G$ with $n \geq 2$ is not stable if and only if for some $v$, all the components of $G-v$ are single vertices, which holds if and only if $v$ is adjacent to all other vertices of $G$, i. e., $G=K_{1, n-1}$. For $n=2,3$, the only tree is $K_{1, n-1}$, and for $n=1$, the only tree is $K_{1}$.)

Any graph $G=K_{2 k}-E\left(C_{2 k}\right)$ is a stable graph, since the consecutive vertices of $C_{2 k}$ colored as $1,1,2,2, \ldots, k, k$ give a coloring of $G$ and, for $G-v=$ $K_{2 k-1}-E\left(P_{2 k-1}\right)$, the consecutive vertices of $P_{2 k-1}$ colored as $1,1,2,2, \ldots, k-$ $1, k-1, k$ give a coloring of $G-v$. 
The following theorem characterizes the stable graphs having chromatic number three.

Theorem 9 Suppose $\chi(G)=3$. Then $G$ is stable if and only if no vertex is in all odd cycles.

Proof: Suppose $\chi(G)=3$. Then $\chi(G-v)=3$ for any $v$ in $V$ if and only if $G-v$ is not bipartite for any $v$ in $V$ if and only if $G-v$ has an odd cycle for any $v$ in $V$ if and only if no vertex $v$ is in all odd cycles.

Thus, the Petersen graph (Figure 5.1) is stable by Theorem 9, since it has chromatic number three and has two vertex-disjoint 5-cycles-the outer, pentagon-shaped cycle and the inner, star-shaped cycle.

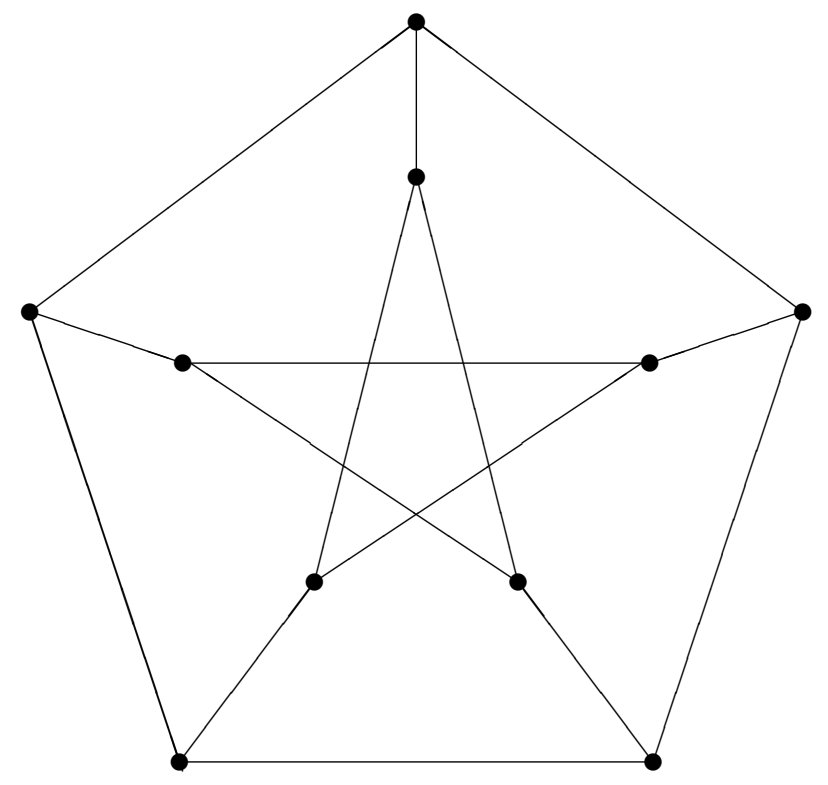

Figure 5.1: Petersen Graph

The following lemma is useful in proving properties of stable graphs. 
Lemma 1 A graph $G$ is stable if and only if for any $\chi(G)$-coloring of $G$, every color class has at least two vertices.

Proof: If some $\chi(G)$-coloring $c$ of $G$ has a color class with only one vertex $v$, then $\chi(G-v)=\chi(G)-1$ as shown by the $(\chi(G)-1)$-coloring of $c$ restricted to $G-v$, and so $G$ is not stable. Conversely, if $G$ is not stable, then $\chi(G-v)=\chi(G)-1$ for some vertex $v$. Then any $(\chi(G)-1)$-coloring of $G-v$ combined with a new color for $v$ yields a $\chi(G)$-coloring of $G$ having a color class $\{v\}$ with only one vertex.

Theorem 10 Suppose $G$ is a stable graph with $n$ vertices. Then $\chi(G) \leq$ $\lfloor n / 2\rfloor$.

Proof: By Lemma 1, for a $\chi(G)$-coloring of $G$, any color class has at least two vertices. Thus, $n \geq 2 \chi(G)$, so that $\chi(G) \leq\lfloor n / 2\rfloor$.

For any $k \geq 2$, we may easily construct infinitely many stable graphs $G$ with $\chi(G)=k$, as shown by the following theorem.

Theorem 11 A graph $G$ is stable if $G$ has vertex-disjoint subgraphs $H_{1}$ and $H_{2}$ with $\chi\left(H_{1}\right)=\chi\left(H_{2}\right)=\chi(G)$.

Proof: For any vertex $v, \chi(G-v) \geq \chi\left(H_{i}\right)=\chi(G)$, where $H_{i}$ does not contain $v$. Hence, $\chi(G-v)=\chi(G)$, i. e., $G$ is stable.

Note that Theorem 11 also suffices to prove (with $H_{1}$ and $H_{2}$ as 5-cycles) that the Petersen graph is stable.

Thus, Theorem 11 shows that joining two vertex-disjoint graphs of chromatic number $k$ with edges (an edge possible between any pair of vertices having different colors) always forms a stable graph $G$ with $\chi(G)=k$ if $k \geq 2$. Hence, if the asterisk is used to denote such a joining of graphs, then if $G_{1}$ is stable, $G_{i+1}=G_{i} * G_{i}, i \geq 1$, is an infinite class of graphs with each $\chi\left(G_{i}\right)=\chi\left(G_{1}\right)$. 
The converse of Theorem 11 is not true, as shown for $\chi(G)=3$ by the following example. The graph $G_{1}$ contains the triangle $v_{1} v_{2} v_{3}$ (colored 1,2,3) with $v_{4}$ (colored 3 ) adjacent to $v_{1}$ and $v_{2}, v_{5}$ (colored 1) adjacent to $v_{2}$ and $v_{3}$, and $v_{6}$ (colored 2) adjacent to $v_{1}$ and $v_{3}$ (Figure 5.2).

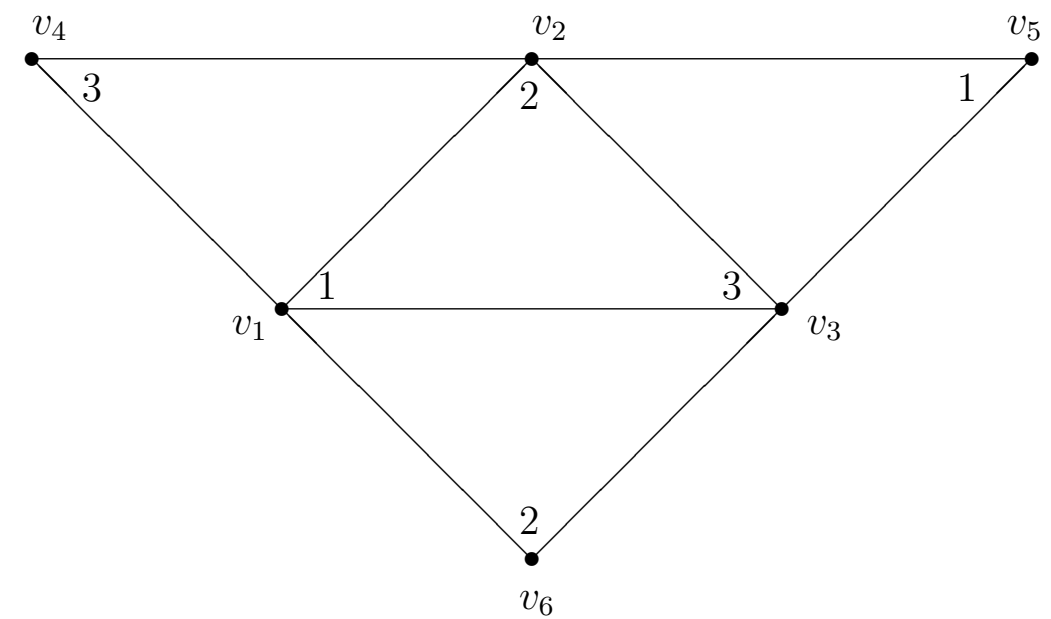

Figure 5.2: $G_{1}$

Since no vertex is in all triangles, then $G_{1}$ is stable by Theorem 9. Since no two triangles of $G_{1}$ are vertex disjoint, then the converse of Theorem 11 does not hold for $G_{1}$.

\subsection{Dynamic Colorings}

We now consider stable graphs for dynamic colorings.

Examples of stable graphs (for $\chi_{d}(G)=3$ ) are $C_{n}$ if and only if $n=3 j$, $j \geq 2$, and any tree (other than $K_{1, n-1}$ or the tree obtained by subdividing $j$ edges of $K_{1, n-1-j}$ for any $j \leq\lfloor(n-1) / 2\rfloor$ ) if and only if $n \geq 6$. (Since any tree of at least three vertices has dynamic chromatic number three, then a tree with $n \geq 3$ is not stable if and only if for some $v, G-v$ has no components other than $K_{1}$ and $K_{2}$, i.e., if and only if $G=K_{1, n-1}$ or $G$ is obtained by 
subdividing some of the edges in $K_{1, i}$. Since any tree with $3 \leq n \leq 5$ is easily seen to be of the excluded form, and since the only tree for $n=1,2$ is $K_{n}$, then the assertion is true as stated.)

Any graph $G=K_{2 k}-E\left(C_{2 k}\right)$ for $k=\chi_{d}(G) \geq 3$ is a stable graph, since the proper colorings given for $G$ and $G-v$ are also dynamic colorings.

A stable graph with $\chi_{d}(G) \geq 4, \chi_{d}(G) \neq \chi(G)$, is obtained by joining two vertex-disjoint graphs $C_{4}$ with an edge $\left(\chi_{d}(G)=4, \chi(G)=2\right)$.

The proof of Theorem 9 uses facts of regular colorings that are not true for dynamic colorings. Both implications of the theorem are false for dynamic colorings: $\chi_{d}\left(G_{1}-v_{5}-v_{6}\right)=3$ and $G_{1}-v_{5}-v_{6}$ is stable, but $v_{1}$ is in all odd cycles; $\chi_{d}\left(G_{2}\right)=3$ and no vertex is in all odd cycles, but $G_{2}$ is not stable, since $\chi_{d}\left(G_{2}-v\right)=\chi_{d}\left(C_{5}\right)=5$ (Figure 5.3).

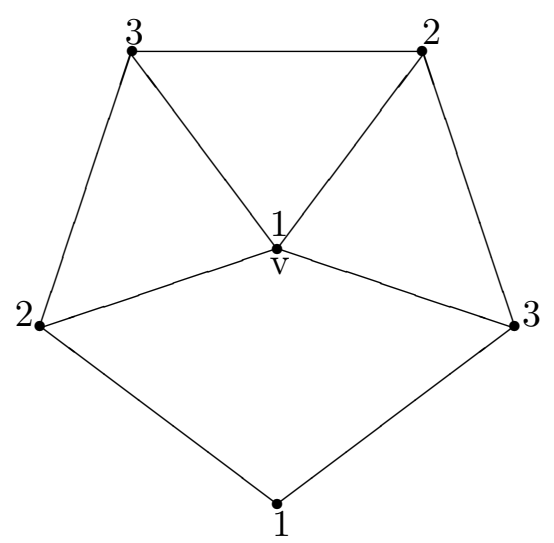

Figure 5.3: $G_{2}$

Because a dynamic coloring of $G$ need not be a dynamic coloring of $G-v$, then the proof of Lemma 1 does not hold for dynamic colorings. In fact, Lemma 1 and Theorem 10 (which was proved using Lemma 1) are false for dynamic colorings, as shown by the following examples. The diamond graph $G_{1}-v_{5}-v_{6}$ and $G_{1}-v_{6}$ are each dynamic stable graphs with the colorings given for $G_{1}$. Each coloring has the color class for the color 2 equal to $\left\{v_{2}\right\}$. 
Also, $\chi_{d}\left(G_{1}-v_{5}-v_{6}\right)=3>2=\left\lfloor\frac{4}{2}\right\rfloor$ and $\chi_{d}\left(G_{1}-v_{6}\right)=3>2=\left\lfloor\frac{5}{2}\right\rfloor$.

Examples are known of dynamic stable graphs with $\chi_{d}(G) \geq 4$ and having a color class with only one vertex. But these examples satisfy $\chi_{d}(G) \leq\lfloor n / 2\rfloor$, and so whether $\chi_{d}(G) \leq\lfloor n / 2\rfloor$ for dynamic stable graphs with $\chi_{d}(G) \geq 4$ remains a topic of investigation.

Theorem 11 is also false for dynamic colorings, as shown by the graph $G_{3}$ (Figure 5.4). The subgraphs $H_{1}=G_{3}\left(v_{1}, v_{2}, v_{3}, v_{4}\right)$ and $H_{2}=G_{3}\left(v_{5}, v_{6}, v_{7}\right)$ are each paths, which have 3 -colorings identical to the one shown for $G_{3}$. However, $\chi_{d}\left(G_{3}-v_{1}\right)=4$ (change the color for $v_{3}$ to 4 ), so that $G_{3}$ is not a stable graph.

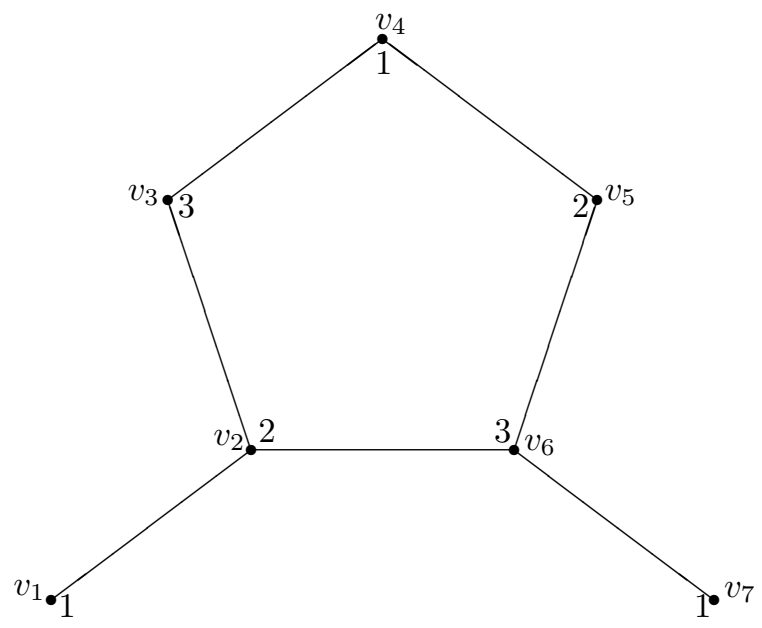

Figure 5.4: $G_{3}$ 


\section{Chapter 6}

\section{Comparison of $\chi$ and $\chi_{d}$}

A natural question to ask is how much the difference between $\chi_{d}(G)$ and $\chi(G)$ varies, and the problem is investigated in this chapter. Graphs for which the two chromatic numbers are identical are the subject of the first section. The second section investigates whether the difference is bounded for all graphs. The final two sections consider classes of graphs for which the difference is small between any two graphs in the class.

\subsection{Normal Graphs}

A graph $G$ is defined to be normal if $\chi_{d}(G)=\chi(G)$.

If $n$ is odd and a multiple of three, then $C_{n}$ is normal; any other cycle is not normal. Any complete graph is normal. The only trees that are normal are $K_{1}$ and $K_{2}$.

Theorem 12 If any vertex of degree greater than one is in a triangle, then $G$ is normal.

Proof: If a vertex is in a triangle, then its two neighbors in the triangle are adjacent and by the adjacency condition must be colored differently in 
any proper coloring of $G$. Thus, any proper coloring of $G$ is also a dynamic coloring of $G$, and so $\chi_{d}(G)=\chi(G)$.

The condition presented in Theorem 12, while sufficient for a graph to be normal, is not necessary. This is demonstrated by the following theorem, in which a method used to construct triangle-free graphs ([2], Theorem 8.7, page 129) is shown to also produce normal graphs when the initial graph is a normal graph.

Theorem 13 For every $k \geq 1$, there exists a normal, triangle-free, $k$-chromatic graph.

Proof: Let $G_{k}$ denote the normal, triangle-free, $k$-chromatic graph. Let $G_{1}=K_{1}, G_{2}=K_{2}$, and $G_{3}=C_{9}$.

Construct $G_{k+1}$ from $G_{k}$ by adding $n+1$ vertices $\left\{u_{1}, \ldots, u_{n}, v\right\}$ to the vertices $\left\{v_{1}, \ldots, v_{n}\right\}$ of $G_{k}$ and by joining $u_{i}$ to each vertex $v_{j}$ to which $v_{i}$ is adjacent; $v$ is joined to each $u_{i}$.

Assume a proper $k$-coloring of $G_{k}$ is given. Then color $u_{i}$ the same as $v_{i}$ and color $v$ a $(k+1)$ st color. Then the proof that $G_{k+1}$ is triangle free and $\chi\left(G_{k+1}\right)=k+1$ is the same as the proof given in [2] when $G_{3}=C_{5}$, since $C_{9}$ is also triangle free $\left(G_{k+1}\right.$ clearly has no triangles. For, since $\left\{u_{1}, u_{2}, \ldots, u_{n}\right\}$ is an independent set in $G_{k+1}$, no triangles can contain more than one $u_{i}$; and if $u_{i} v_{j} v_{k} u_{i}$ were a triangle in $G_{k+1}$, then $v_{i} v_{j} v_{k} v_{i}$ would be a triangle in $G_{k}$, contrary to assumption. We now show that $G_{k+1}$ is $(k+1)$-chromatic. Note, first, that $G_{k+1}$ is certainly $(k+1)$-colorable, since any $k$-coloring of $G_{k}$ can be extended to a $(k+1)$-coloring of $G_{k+1}$ as described above. Therefore it remains to show $G_{k+1}$ is not $k$-colorable. If possible, consider a $k$-coloring of $G_{k+1}$ in which, without loss of generality, $v$ is assigned color $k$. Clearly, no $u_{i}$ can also have color $k$. Now recolor each vertex $v_{i}$ of color $k$ with the color assigned to $u_{i}$. This results in a $(k-1)$-coloring of the $k$-chromatic graph $G_{k}$. Therefore $G_{k+1}$ is indeed $(k+1)$-chromatic.) 
Suppose that each vertex $v_{i}$ of $G_{k}$ has some neighbors of different colors, where $k \geq 3$. Since the neighbors of $v_{i}$ are also neighbors of $u_{i}$, then $u_{i}$ has some neighbors of different colors in $G_{k+1}$. Since each $u_{i}$ is colored the same as $v_{i}$, which are not all colored the same, then $v$, being adjacent to each $u_{i}$, has some neighbors of different colors in $G_{k+1}$. Thus, by using a dynamic coloring of $G_{3}=C_{9}$, the $k$-coloring of $G_{k}$ is guaranteed to satisfy the doubleadjacency condition for all $k \geq 3$. Since $\chi_{d}\left(C_{9}\right)=\chi\left(C_{9}\right)$, then by induction $\chi_{d}\left(G_{k}\right)=\chi\left(G_{k}\right)=k$ for $k \geq 3$.

Theorem 14 Let $n \geq 3$. If $\delta(G)>\lfloor n / 2\rfloor$, then $G$ is normal. This bound on $\delta$ cannot be lowered.

Proof: Suppose $n \geq 3$. For any vertex $v$, a neighbor of $v$ not adjacent to another neighbor of $v$ would be adjacent to at most $n-\delta(G) \leq\lceil n / 2\rceil-1<$ $\delta(G)$ vertices. Thus, any two adjacent vertices are in a triangle. Hence, by Theorem 12, $G$ is normal.

Let $K_{n_{1}, n_{2}}$ denote the complete bipartite graph, defined as having vertex set $V$ partitioned as $X_{1} \cup X_{2},\left|X_{i}\right|=n_{i}$ for $i=1,2$, and having any vertex of $X_{1}$ adjacent to any vertex of $X_{2}$ as its vertex adjacencies. Let $G=K_{\lfloor n / 2\rfloor,\lceil n / 2\rceil}$ for $n \geq 3$. Then $\delta(G)=\lfloor n / 2\rfloor$. Since $\chi(G)=2$ and $\chi_{d}(G) \geq 3$, then $G$ is not normal.

\section{2 $\chi_{d}-\chi$ is Unbounded}

In this section, we consider whether there exists a constant upper bound for $\chi_{d}(G)-\chi(G)$ that holds for all graphs $G$.

One class of graphs important in showing the difference between $\chi_{d}(G)$ and $\chi(G)$ is the class $S K_{k}$ of bipartite graphs defined as follows for $k \geq 3$. The graph $S K_{k}$ has vertex set $V$ partitioned as $X \cup Y$, where $|X|=k$ and 
$|Y|=\left(\begin{array}{c}k \\ 2\end{array}\right)$. Each vertex in $Y$ is adjacent to two vertices in $X$, and distinct vertices in $Y$ are adjacent to distinct pairs of vertices in $X$. Thus, $d(v)=k-1$ for each $v \in X$. Note that $S K_{k}$ may be obtained from $K_{k}$ by subdividing each edge of $K_{k}$. Then $X=V\left(K_{k}\right)$ and $Y$ is the set of vertices used to subdivide the edges.

In a dynamic coloring of $S K_{k}$, any two vertices of $X$ must be assigned different colors, since otherwise some vertex of $Y$ would be adjacent to exactly two vertices, both assigned the same color. Hence, $\chi_{d}\left(S K_{k}\right) \geq k$. If $X=$ $\left\{x_{1}, \ldots, x_{k}\right\}$, then the coloring $c\left(x_{i}\right)=i, c(y) \in\{1, \ldots, k\}$ and $c(y) \neq i, j$ if $y \in Y$ is adjacent to $x_{i}$ and $x_{j}$, is a dynamic coloring of $S K_{k}$. Thus, $\chi_{d}\left(S K_{k}\right)=k$.

Thus, the difference between $\chi_{d}$ and $\chi$ is unbounded.

\section{3 $\quad K_{1,3}$-Free Graphs}

For $k \geq 4, S K_{k}$ contains $K_{1,3}$, one of the smallest and simplest graphs $G$ for which $\chi_{d}(G)$ and $\chi(G)$ differ, as an induced subgraph at each vertex of $V$. This suggests considering $K_{1,3}$-free graphs as a possible class of graphs for which $\chi_{d}(G)-\chi(G)$ is bounded.

Lemma 2 Suppose $G$ is connected and $G$ is $K_{1,3}$-free. If $\chi(G)=2$, then $\chi_{d}(G) \leq 4$ with $\chi_{d}(G)=4$ only if $G$ is a cycle of even length not a multiple of 3 .

Proof: Suppose $\chi(G)=2$ and $G$ is $K_{1,3}$-free. Then $\Delta(G) \leq 2$, since otherwise any vertex of degree at least 3 is contained in $K_{3}$, and so $\chi(G) \geq 3$.

If each vertex has degree 2 , then $G$ is an even cycle, since $\chi(G)=2$. Thus, $\chi_{d}(G) \leq 4$, and so $\chi_{d}(G)=4$ only if the cycle also has length not a multiple of 3 . 
Otherwise, each vertex has degree 1 or degree 2 , so that $G$ is a path. Thus, $\chi_{d}(G)=2$ or $\chi_{d}(G)=3$.

The length of a cycle is the number of vertices in the cycle, and the length of a path is one less than the number of vertices in the path.

Theorem 15 If $G$ is $K_{1,3}$-free, then $\chi_{d}(G) \leq \chi(G)+2$, and equality holds if and only if $G$ is a cycle of length 5 or of even length not a multiple of 3.

Proof: The upper bound holds as stated for any cycle $C_{n}$.

Assume henceforth that $G$ is not a cycle.

Define an arc to be a path for which all the internal vertices have degree two. Let $l$ denote the maximum length of an arc in $G$, and let $u$ and $v$ typically denote the endvertices of such an arc $P_{u, v}$. Let $G^{\prime}$ denote the subgraph of $G$ induced by $V(G)-V\left(P_{u, v}\right) \cup\{u, v\}$. Let the neighborhood $N_{u}$ of $u$ be the subgraph of $G$ induced by $u$ and its adjacent vertices in $G^{\prime}$. Since $G$ is $K_{1,3}$-free, then if $l \geq 3, N_{u}$ and $N_{v}$ are complete, which must also hold for $l=2$ if $u$ and $v$ are nonadjacent.

The proof is by induction on $n$. The result is easily verified for $n \leq 3$.

Suppose $l=1$. Then $G$ has no arcs of length at least two and hence no vertices of degree two. Thus, any vertex of degree greater than one is in some $K_{3}$, since $G$ is $K_{1,3}$-free. Hence, any proper coloring is a dynamic coloring, and so $\chi_{d}(G)=\chi(G)$.

Suppose $l=2$.

If $\chi\left(G^{\prime}\right)=1$, then $G^{\prime}$ consists of the disjoint vertices $u$ and $v$, so that $G=P_{u v}$ and $\chi_{d}(G)=3=\chi(G)+1$.

Suppose $\chi\left(G^{\prime}\right)=2$. If $G^{\prime}$ is not connected, then its two components, if nontrivial, could by the lemma only be paths or even cycles, which would violate $G K_{1,3}$-free (at $u$ or $v$.) So, $G=P_{u, v}$. If $G^{\prime}$ is connected, $G^{\prime}$ is a path or even cycle. Since $G$ is $K_{1,3}$-free, then $u$ and $v$ are adjacent in the path 
that is $G^{\prime}$, and the path may have $u$ or $v$ as an endvertex or may extend one or two vertices past $u$ or $v$. Then $\chi_{d}(G)=\chi(G)=3$.

Suppose $\chi\left(G^{\prime}\right) \geq 3$. Hence, $\chi\left(G^{\prime}\right)=\chi(G)$.

Suppose some optimal dynamic coloring $c$ of $G^{\prime}$ has $c(u) \neq c(v)$. Then $\chi_{d}(G)=\chi_{d}\left(G^{\prime}\right)$. Since $l=2, G^{\prime}$ is not a cycle of length greater than three, hence also not an even cycle. Thus, $\chi_{d}(G)=\chi_{d}\left(G^{\prime}\right) \leq \chi\left(G^{\prime}\right)+1=\chi(G)+1$, so that $\chi_{d}(G) \leq \chi(G)+1$.

Suppose any optimal dynamic coloring $c$ of $G^{\prime}$ has $c(u)=c(v)$. Since $N(u), N(v)$ are complete subgraphs and $|N(u)| \geq 3$ or $|N(v)| \geq 3$, then $|N(u)|=\chi_{d}\left(G^{\prime}\right)$ or $|N(v)|=\chi_{d}\left(G^{\prime}\right)$, since otherwise $G^{\prime}$ could be recolored by recoloring $u$ to be in $c\left(G^{\prime}\right)-c(N(u))$ or, respectively, by recoloring $v$ to be in $c\left(G^{\prime}\right)-c(N(v))$, yielding an optimal dynamic coloring $c^{\prime}$ of $G^{\prime}$ having $c^{\prime}(u) \neq c^{\prime}(v)$. Thus, $\chi_{d}\left(G^{\prime}\right)=\omega\left(G^{\prime}\right)$ and, since $\omega \leq \chi \leq \chi_{d}$ for any graph, then $\chi_{d}\left(G^{\prime}\right)=\chi\left(G^{\prime}\right)$. Since $\chi_{d}(G) \leq \chi_{d}\left(G^{\prime}\right)+1$, then $\chi_{d}(G) \leq \chi\left(G^{\prime}\right)+1=$ $\chi(G)+1$.

Suppose $l \geq 3$.

Suppose $\chi_{d}\left(G^{\prime}\right) \leq 3$. Since the remaining vertices of $P_{u v}$ may be colored with four colors including $c\left(G^{\prime}\right)$ to extend any optimal dynamic coloring $c$ of $G^{\prime}$ to a dynamic 4-coloring of $G$, then $\chi_{d}(G) \leq 4$. By Lemma 15, $\chi(G)=2$ when $\chi_{d}(G)=4$ only if $\mathrm{G}$ is a cycle of even length not a multiple of three.

Suppose $\chi_{d}\left(G^{\prime}\right) \geq 4$. Then $\chi\left(G^{\prime}\right)=1$ is not possible, since then $G=$ $P_{u v}$ and $\chi_{d}\left(P_{u v}\right)=3$. Consider $\chi\left(G^{\prime}\right)=2$. If not connected, $G^{\prime}$ has two components which, by the lemma, would be incomplete graphs if nontrivial; $G=P_{u v}$ if trivial. If $G^{\prime}$ is connected and hence a path of length at least three or a cycle of length at least four, then $N_{u}$ and $N_{v}$ are incomplete.

Consider $\chi\left(G^{\prime}\right) \geq 3$. Then $\chi(G)=\chi\left(G^{\prime}\right)$. Also, $\chi_{d}(G) \leq \chi_{d}\left(G^{\prime}\right)$, since any dynamic coloring $c$ of $G^{\prime}$ can be extended to a dynamic coloring of $G$ by coloring the remaining vertices of $P_{u v}$ with colors of $c\left(G^{\prime}\right)$ so that at most four colors of $c\left(G^{\prime}\right)$ color $P_{u v}$. If $G^{\prime}$ is a cycle, then $G^{\prime}=K_{3}$ to ensure $N_{u}$ 
and $N_{v}$ are complete; in this case, $\chi_{d}(G)=4$ and $\chi(G)=3$. Otherwise, $\chi_{d}\left(G^{\prime}\right) \leq \chi\left(G^{\prime}\right)+1$ by the induction hypothesis. Thus, $\chi_{d}(G) \leq \chi_{d}\left(G^{\prime}\right) \leq$ $\chi\left(G^{\prime}\right)+1=\chi(G)+1$, and so $\chi_{d}(G) \leq \chi(G)+1$.

\subsection{Regular Graphs}

A graph is said to be $k$-regular if each vertex of $G$ has degree $k$. A regular graph is a graph that is $k$-regular for some $k \geq 1$.

Because of this uniformity of vertex degree and the symmetry it may cause in a graph, it is conjectured that $\chi_{d}(G)-\chi(G)$ is typically small for regular graphs. In fact, we conjecture that $\chi_{d}(G)-\chi(G) \leq 2$ for any regular graph.

If $k>\lfloor n / 2\rfloor$, then $\delta>\lfloor n / 2\rfloor$, and thus $\chi_{d}(G)-\chi(G)=0$ is Theorem 14 previously shown.

If $k=1$, then $G=K_{2}$, and $\chi_{d}(G)-\chi(G)=2-2=0$.

If $k=2$, then $G$ is a cycle, and $\chi_{d}(G)-\chi(G)=0,1$, or 2 for any cycle, as previously shown.

If $k=3$, then $\chi_{d}(G) \leq 5$ by Theorem 5 , and so $\chi_{d}(G)-\chi(G) \leq 2$ except possibly when $G$ is bipartite.

The Petersen graph is a 3-regular graph, and $\chi_{d}(G)-\chi(G)=4-3=1$.

We now investigate the case of $k$-regular bipartite graphs.

Theorem 16 Suppose $G$ is a $k$-regular bipartite graph for $k \geq 3$. If $n<2^{k}$, then $\chi_{d}(G) \leq 4$.

Proof: Since $G$ is bipartite and $k$-regular, then $V(G)=(U, W)$ and $|U|=$ $|W|=n / 2$. Let $m=n / 2, U=\left\{u_{1}, \ldots, u_{m}\right\}$, and $W=\left\{w_{1}, \ldots, w_{m}\right\}$.

For any particular $i, 1 \leq i \leq m, N\left(w_{i}\right)$ is contained in $\left(\begin{array}{c}m-k \\ \lceil m / 2\rceil-k\end{array}\right)$ 
$\lceil m / 2\rceil$-subsets $S$ of $U$. Thus, there are at most $m\left(\begin{array}{c}m-k \\ \lceil m / 2\rceil-k\end{array}\right)\lceil m / 2\rceil$ subsets $S$ of $U$ that contain at least one $N\left(w_{i}\right), 1 \leq i \leq m$, so that there are at most $2 m\left(\begin{array}{c}m-k \\ \lceil m / 2\rceil-k\end{array}\right)\lceil m / 2\rceil$-subsets $S$ of $U$ such that $S$ or $U-S$ contains at least one $N\left(w_{i}\right)$.

$$
\begin{array}{r}
\text { Since } 2 m\left(\begin{array}{c}
m-k \\
\lceil m / 2\rceil-k
\end{array}\right)<\left(\begin{array}{c}
m \\
\lceil m / 2\rceil
\end{array}\right) \text { if and only if } \\
\frac{2 m(m-k) !}{\lfloor m / 2\rfloor !(\lceil m / 2\rceil-k) !}<\frac{m !}{\lfloor m / 2\rfloor !\lceil m / 2\rceil !}
\end{array}
$$

if and only if

$$
2 m<\frac{m(m-1) \cdots(m-k+1)}{\lceil m / 2\rceil\lceil m / 2\rceil(\lceil m / 2\rceil-1) \cdots(\lceil m / 2\rceil-k+1)}
$$

if $n<2^{k}$, then if $n<2^{k}$, there is a $\lceil m / 2\rceil$-subset $S$ of $U$ such that both $S$ and $U-S$ contain no $N\left(w_{i}\right)$. Similarly, if $n<2^{k}$, there is a $\lceil m / 2\rceil$-subset $T$ of $W$ such that both $T$ and $W-T$ contain no $N\left(u_{i}\right)$.

Hence, if each vertex in $S, U-S, T$, or $W-T$ is colored $1,2,3$, or 4 , respectively, then the double-adjacency condition is satisfied, since no $w_{i}$ is adjacent only to vertices colored 1 or only to vertices colored 2 and no $u_{i}$ is adjacent only to vertices colored 3 or only to vertices colored 4 . The adjacency condition is satisfied, since $G$ is bipartite and partition sets $U$ and $W$ are colored differently. 


\section{Chapter 7}

\section{Graphs with $\chi_{d}(G)=n$ or $n-1$}

We first consider graphs for which the chromatic number equals $n$ or $n-1$, and then consider the graphs for which the dynamic chromatic number equals $n$ or $n-1$.

There are only two (limited) types of graphs for which the chromatic number equals $n$ or $n-1$, which is now discussed.

If two vertices of $G$ are nonadjacent, then they may be colored alike in an $(n-1)$-coloring of $G$; hence $\chi(G)=n$ only for $G=K_{n}$.

Suppose $\chi(G)=n-1$. Then $\bar{G}$ has no clique $H=K_{j}$ of $j \geq 3$ vertices, since then $G$ has a proper (not necessarily minimal) coloring $c$ with $|c(V(H))|=1$, showing $\chi(G) \leq|c(V(G))| \leq n-2$. Also, $\bar{G}$ has no "parallel edges" (i. e., $x_{1} x_{2}, y_{1} y_{2} \in E(V(\bar{G}))$ and $\left|\left\{x_{1}, x_{2}, y_{1}, y_{2}\right\}\right|=4$ ), since then $G$ has a proper (not necessarily minimal) coloring $c$ with $c\left(x_{1}\right)=c\left(x_{2}\right)$, $c\left(y_{1}\right)=c\left(y_{2}\right)$, showing $\chi(G) \leq|c(V(G))| \leq n-2$.

Thus, $\bar{G}$ has no cliques of three or more vertices and $\bar{G}$ has no parallel edges. Hence, $\bar{G}$ has no edges (impossible, since then $G=K_{n}$ ) or all the edges of $\bar{G}$ have a common vertex $v$ but otherwise any pair of edges in $\bar{G}$ is nonadjacent (i. e., the subgraph of edges in $\bar{G}$ is $K_{1, j}$ for some $j \geq 1$, yet $j \leq n-2$ for $v$ to not be an isolated vertex in the connected graph $G$ ). So, 
$G=K_{n}-E\left(K_{1, j}\right)=K_{n}-v u_{1}-\ldots-v u_{j}$ for any $1 \leq j \leq n-2$, and the $(n-1)$-colorings of $G$ are the colorings $c_{i}, 1 \leq i \leq j$, with $c_{i}(v)=c_{i}\left(u_{i}\right)$ and otherwise $c_{i}(x) \neq c_{i}(y)$ if $x \neq y$, i. e., $\left\{v, u_{i}\right\}$ is the only color class of $c_{i}$ of two or more vertices.

We now investigate the graphs for which the dynamic chromatic number equals $n$ or $n-1$, and see that this set of graphs contains those just obtained but is more extensive and complex.

For what graphs must a different color be assigned to each vertex in a minimal coloring? If $n \geq 2$, then $G \subset K_{n}-e$ for an edge $e$ implies $\chi(G) \leq$ $\chi\left(K_{n}-e\right)=n-1$. But such reasoning is not valid for dynamic colorings, since $H \subset G$ does not imply $\chi_{d}(H) \leq \chi_{d}(G)$ (for example, $\chi_{d}\left(C_{5}\right)=5>4=$ $\chi_{d}(G)$ if $G$ consists of $C_{5}$ and an additional vertex adjacent to one vertex of $\left.C_{5}\right)$.

Lemma 3 Suppose $\chi_{d}(G)=n$. Then if $u$ and $v$ are not neighbors, $u$ and $v$ are the only neighbors of some vertex $x$ (i.e., $u \notin N(v)$ implies $N(x)=\{u, v\}$ for some $x \neq u, v)$.

Proof: Since $u$ and $v$ are nonadjacent vertices, an $(n-1)$-coloring of $G$ in which only $u$ and $v$ receive the same color must satisfy both the adjacency and double-adjacency conditions unless the double-adjacency condition is not satisfied for some vertex $x$ adjacent to $u$ and $v$.

We denote the path of $n$ vertices by $P_{n}$.

Theorem 17 The dynamic chromatic number $\chi_{d}(G)=n$ if and only if $G=$ $P_{3}, C_{4}, C_{5}$, or $K_{n}$.

Proof: The theorem is clearly true for $n \leq 3$, and the specified graphs satisfy $\chi_{d}(G)=n$ for any $n$.

Suppose $n \geq 4$ and $\chi_{d}(G)=n$. If $G \neq K_{n}$, suppose $u_{1}$ and $u_{2}$ are not neighbors. Hence, by Lemma 3, $N\left(v_{1}\right)=\left\{u_{1}, u_{2}\right\}$ for some $v_{1} \neq u_{1}, u_{2}$. Since 
$n \geq 4$, then $v_{1} \notin N\left(v_{2}\right)$ for some vertex $v_{2} \neq u_{1}, u_{2}, v_{1}$. Hence, by Lemma 3, $N(w)=\left\{v_{1}, v_{2}\right\}$ for some $w \neq v_{1}, v_{2}$. Since $N\left(v_{1}\right)=\left\{u_{1}, u_{2}\right\}$, then $w=u_{1}$ or $w=u_{2}$, say $w=u_{2}$ (without loss of generality), which gives $N\left(u_{2}\right)=\left\{v_{1}, v_{2}\right\}$

If $u_{1} v_{2} \in E(G)$, then if $n=4$, all possible edges of the graph have been determined, so that $G=u_{1} v_{1} u_{2} v_{2} u_{1}=C_{4}$. If $n>4$, then since $N\left(v_{1}\right)=$ $\left\{u_{1}, u_{2}\right\}, v_{1} \notin N(x)$ for any $x \neq u_{1}, u_{2}, v_{2}$. Hence, by Lemma 3, $N(y)=$ $\left\{v_{1}, x\right\}$ for some $y \neq v_{1}, x$. Since $N\left(v_{1}\right)=\left\{u_{1}, u_{2}\right\}$, then $y=u_{1}$ or $y=u_{2}$, which is impossible since $v_{2} \in N\left(u_{1}\right)$ and $v_{2} \in N\left(u_{2}\right)$ (but $x \neq v_{2}$ ).

If $u_{1} v_{2} \notin E(G)$, then $N(z)=\left\{u_{1}, v_{2}\right\}$ for some $z \neq u_{1}, v_{2}$. Also, $z \neq$ $v_{1}, u_{2}$, since $v_{1} u_{2} \in E(G)$. If $n=5$, then all possible edges have been determined, so that $G=u_{1} v_{1} u_{2} v_{2} z u_{1}=C_{5}$. If $n \geq 6$, then since $N\left(v_{1}\right)=$ $\left\{u_{1}, u_{2}\right\}, v_{1} \notin N(x)$ for any $x \neq u_{1}, u_{2}, v_{2}, z$. Hence, by Lemma 3, $N(y)=$ $\left\{v_{1}, x\right\}$ for some $y \neq v_{1}, x$. Since $N\left(v_{1}\right)=\left\{u_{1}, u_{2}\right\}$, then $y=u_{1}$ or $y=u_{2}$, which is impossible, since $z \in N\left(u_{1}\right)$ but $z \neq v_{1}, x$, and since $v_{2} \in N\left(u_{2}\right)$ but $v_{2} \neq v_{1}, x$.

Now consider the case $\chi_{d}(G)=n-1$. Two lemmas are useful. The first follows directly from a result in [4] showing that the upper bound of $\chi_{d}(G) \leq \Delta+2$ (if $G \neq C_{5}$ ) in Theorem 5 is achieved with equality only for certain cycles.

Lemma 4 If $\Delta=2$, then $\chi_{d}(G) \leq 5$; if $\Delta \geq 3$, then $\chi_{d}(G) \leq \Delta+1$.

Lemma 5 Suppose $\chi_{d}(G)=n-1$. Then $G=P_{4}$ if $\Delta=2$, and $\Delta \geq n-2$ if $\Delta \geq 3$.

Proof: By Lemma $4, \chi_{d}(G) \leq 5$ if $\Delta=2$, so that if $\chi_{d}(G)=n-1$, then $n \leq 6$. The only graph satisfying these conditions is $P_{4}$.

Theorem 18 The dynamic chromatic number $\chi_{d}(G)=n-1$ if and only if $G$ is one of the following graphs: $P_{4} ; K_{n}-E\left(K_{1, j}\right), 1 \leq j \leq n-2$, for $n \geq 4$; $K_{n-1}-x y, n \geq 6$, and an additional vertex adjacent only to $x$ and $y$; or the four graphs shown in Figure 7.1. 
Proof: If $\Delta=1$, then $G=K_{2}$ but $\chi_{d}\left(K_{2}\right)=2$.

If $\Delta=2$, then by Lemma $5, G=P_{4}$ if $\chi_{d}(G)=n-1$. Also, $\chi_{d}\left(P_{4}\right)=$ $3=n-1$.

If $\Delta \geq 3$, then by Lemma $5, \Delta \geq n-2$.

Suppose $\Delta=n-1$. For $n \leq 3$, no graphs satisfy $\chi_{d}(G)=n-1$. Let $n \geq 4$ and let $d(v)=n-1$. Then any proper coloring is easily seen to be a dynamic coloring if $\chi_{d}(G)=n-1$. First, note that $v$ satisfies the doubleadjacency condition since $G \neq K_{1, n-1}$. If $d(x) \geq 2$ and $x \neq v$, then $x y \in E$ for some $y \neq v$ implies $x y v x$ is in $G$, so that $x$ satisfies the double-adjacency condition.

Hence, if $\Delta=n-1$, then since $\chi_{d}(G)=\chi(G)$ for $n \geq 4$, then the graphs that satisfy $\chi_{d}(G)=n-1$ are the graphs that satisfy $\chi(G)=n-1$, i. e., $G=K_{n}-E\left(K_{1, j}\right)$ for some $j$, where $1 \leq j \leq n-2$.

Suppose $\Delta=n-2$. For $n \leq 4$, the only graph $G$ with $\chi_{d}(G)=n-1$ is $G=P_{4}$. So suppose $n \geq 5$. Let $d(v)=n-2$ and let $v^{\prime}$ be the only vertex not adjacent to $v$.

Since $d\left(v^{\prime}\right) \geq 1$, then there is a vertex $x$ adjacent to $v^{\prime}$ that, since $\Delta=$ $n-2$, is not adjacent to some vertex $y \neq v^{\prime}, v$. Thus, a coloring of $G$ with $v, v^{\prime}$ colored alike, $x, y$ colored alike, and otherwise with vertices colored distinctly, is a dynamic coloring of $n-2$ colors unless some vertex $u$ is adjacent only to $x$ and $y$ (or some vertex $u^{\prime}$ is adjacent only to $v$ and $v^{\prime}$ ). Then $u=v^{\prime}$, since any $u \neq v, v^{\prime}$ is adjacent also to $v$. Since $v^{\prime}$ is the only possibility for such a vertex $u$, then $x, y$ is the only such nonadjacent pair of vertices, so that $G$ consists of $K_{n-1}-x y$ and a final vertex $u=v^{\prime}$, which is adjacent precisely to $x$ and $y$.

Suppose some vertex $u^{\prime}$ is adjacent only to $v$ and $v^{\prime}$. Consider first $n=5$. Then the remaining two vertices, $w$ and $w^{\prime}$, are both adjacent to $v$; vertices $u^{\prime}, v$, and $v^{\prime}$ must be colored differently.

If $v^{\prime}$ is not adjacent to $w$ and $w^{\prime}$, then $c(w)=c\left(u^{\prime}\right)$ and $c\left(w^{\prime}\right)=c\left(v^{\prime}\right)$ 
describes a 3-coloring of $G$.

If $v^{\prime}$ is adjacent to at least one of $w$ and $w^{\prime}$, then whichever of $w$ and $w^{\prime}$ is adjacent to $v^{\prime}$ cannot be colored $c(v)$ or $c\left(v^{\prime}\right)$, and cannot be colored $c\left(u^{\prime}\right)$ if it alone is adjacent; also, both $w$ and $w^{\prime}$ cannot be colored $c\left(u^{\prime}\right)$ if both are adjacent to $v^{\prime}$. So, one of $w, w^{\prime}$ must be colored distinctly from $u^{\prime}, v$, and $v^{\prime}$; coloring the other the same as $u^{\prime}$ describes a 4-coloring of $G$. Thus, $\chi_{d}(G)=4$ for these four graphs with $n=5$ (Figure 7.1).
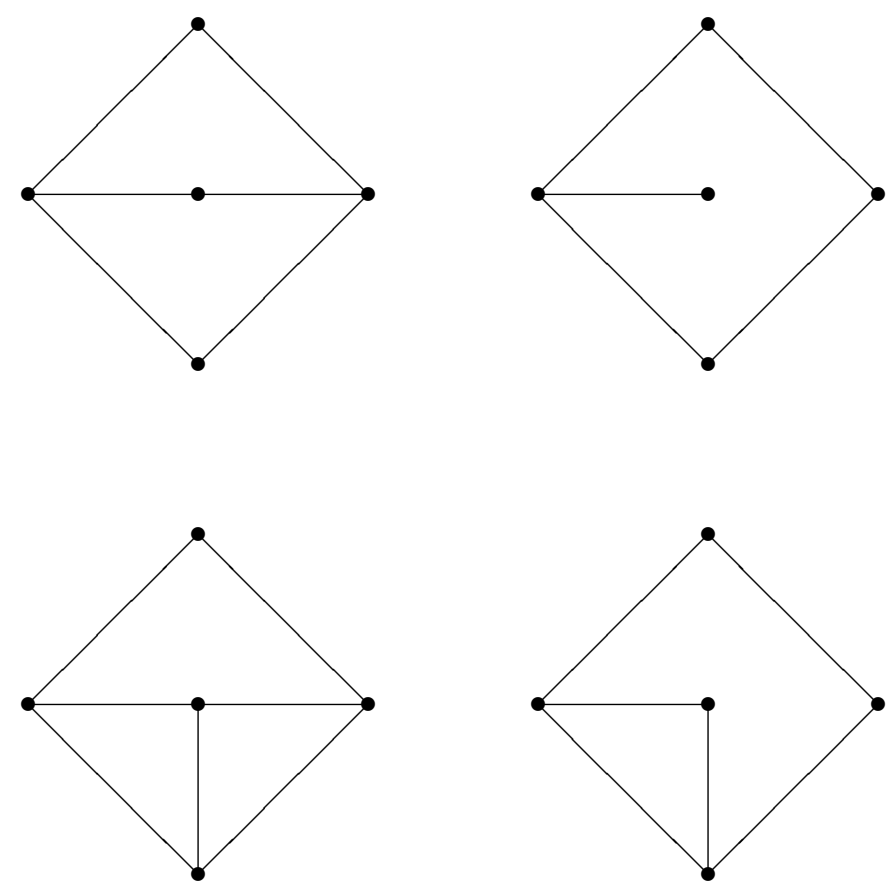

Figure 7.1: $\chi_{d}(G)=n-1$ for $n=5$

Consider $n \geq 6$. We wish to show that $G\left(V-u^{\prime}\right)=K_{n-1}-v v^{\prime}$, where $u^{\prime}$ is adjacent only to $v$ and $v^{\prime}$. Suppose $G\left(V-u^{\prime}\right) \neq K_{n-1}-v v^{\prime}$. Thus, since $v$ is adjacent to every vertex except $v^{\prime}$, we can suppose $G\left(V-\left\{u^{\prime}, v\right\}\right) \neq K_{n-2}$, and consider for $U=V-\left\{u^{\prime}, v, v^{\prime}\right\}$ the two cases $G(U)=K_{n-3}$ and $G(U) \neq K_{n-3}$. 
In each case that follows, the coloring describes an $(n-2)$-dynamic coloring of $G$. All other vertices are colored distinctly.

Suppose $G(U)=K_{n-3}$. Since $v^{\prime}$ is not adjacent to some $w \in U$, then color $w$ the same as $v^{\prime}$ and color another vertex, which is not adjacent to $v^{\prime}$ or is not the only vertex adjacent to $v^{\prime}$, the same color as $u^{\prime}$.

Suppose $G(U) \neq K_{n-3}$. If $v^{\prime}$ is adjacent to no vertex in $U$ or more than one vertex in $U$, color two of the nonadjacent vertices in $U$ the same but differently from $u^{\prime}, v$, and $v^{\prime}$; also color another vertex in $U$ the same as $u^{\prime}$.

If $v^{\prime}$ is adjacent to exactly one vertex $w$ in $\mathrm{U}$, color $w$ differently from $u^{\prime}, v$, and $v^{\prime}$; if $w$ is not adjacent to some $w^{\prime}$ in $U$, then color $w^{\prime}$ the same as $w$ and color another vertex the same as $u^{\prime}$, otherwise color a nonadjacent pair of vertices the same as $u^{\prime}$. 


\section{Chapter 8}

$$
\chi_{d}(G-v)=\chi_{d}(G)-2
$$

For any graph $G, \chi(G-v) \geq \chi(G)-1$ for any vertex $v$ of $G$, since otherwise a $(\chi(G)-2)$-coloring of $G-v$ with an additional color to color $v$ would give a $(\chi(G)-1)$-coloring of $G$.

However, for $G=C_{5}$ or $G=P_{3}, \chi_{d}(G-v)=\chi_{d}(G)-2$ for at least one vertex $v$ of $G$. Thus, it is of interest to know what is the maximum possible decrease in the dynamic chromatic number that can occur with the removal of a vertex, and to know the graphs for which the maximum decrease can occur. These questions are answered completely by the following theorem.

Theorem 19 For any graph $G, \chi_{d}(G-v) \geq \chi_{d}(G)-2$ for any vertex. The only graphs for which $\chi_{d}(G-v)=\chi_{d}(G)-2$ for at least one vertex are $K_{1, n-1}, n \geq 3$, and $C_{5}$.

Proof: Let $c_{1}$ be an optimal dynamic coloring of $G-v$ from $\{1,2, \ldots, m\}$, where $m=\chi_{d}(G-v)$. Let $c_{2}(v)=m+1$ and $c_{2}(x)=c_{1}(x)$ if $x \neq v$. This yields a dynamic coloring $c_{2}$ of $G$ unless $d(v) \geq 2$ and $c_{1}(u)$ is the same for all $u$ in $N(v)$. In that case, $c_{2}$ is defined as before except $c_{2}\left(u_{1}\right)=m+2$ for one vertex $u_{1}$ in $N(v)$ to obtain a dynamic coloring $c_{2}$ of $G$ of $m+2$ colors. Thus, $\chi_{d}(G) \leq m+2=\chi_{d}(G-v)+2$. 
Now an argument is begun to characterize the graphs $G$ for which $\chi_{d}(G-v)=\chi_{d}(G)-2$ for some vertex $v$ of $G$.

Note that, as shown above, if $d(v)=1$ or not all vertices of $N(v)$ are colored the same by some optimal dynamic coloring of $G-v$, then $\chi_{d}(G-v) \geq$ $\chi_{d}(G)-1$. Thus, we assume henceforth that $d(v) \geq 2$ and that $c_{1}(u)=1$ for all $u$ in $N(v)$ for any optimal dynamic coloring $c_{1}$ of $G-v$. This implies that no two vertices of $N(v)$ are adjacent, which is also assumed henceforth.

If $d(u)=1$ for each $u$ in $N(v)$, then $G=K_{1, n-1}, n \geq 3$, which satisfies $\chi_{d}(G-v)=\chi_{d}(G)-2$.

If $d(u)=1$ for some but not all $u$ in $N(v)$, then a coloring $c_{1}$ of $G-v$ with $c_{1}(u)=c_{1}(w)$ for any $u$ in $N(v)$ with $d(u)=1$, where $w \neq v$ is a vertex adjacent to some $u_{1}$ in $N(v)$ with $d\left(u_{1}\right) \geq 2$, has not all vertices in $N(v)$ colored the same.

If $d(u) \geq 3$ for all $u$ in $N(v)$, then since $c_{1}(u)=1$ for all $u$ in $N(v)$, then an $(m+1)$-coloring $c_{2}$ of $G$ is given by specifying for one vertex $u_{1}$ in $N(v)$ that $c_{2}\left(u_{1}\right)=m+1, c_{2}(v)=2$, and $c_{2}(z)=c_{1}(z)$ otherwise.

So assume henceforth that $d(u) \geq 2$ for all $u$ in $N(v)$ and $d(u)=2$ for some $u$ in $N(v)$. Let $U=\{u \in N(v): d(u)=2\}=\left\{u_{i}: 1 \leq i \leq|U|\right\}$ and let $U^{\prime}=\{u \in N(v): d(u) \geq 3\}$. Let $w_{i}$ be the vertex adjacent to $u_{i}$ in $G-v$ and let $W=\left\{w_{i}: 1 \leq i \leq|U|\right\}\left(w_{i}=w_{j}\right.$ if $i \neq j$ is not precluded).

Consider the following condition (to be referenced throughout the remainder of the proof):

There exists $x$, not in $U, U^{\prime}, W$, and adjacent to some $w_{i}$ in $W(*)$

If $\left(^{*}\right)$ holds, then some $w_{i}$ is adjacent to some vertex $x$ not colored 1 , say colored $b \neq 1$. For one such $i$, let $c_{2}(x)=m+1, c_{2}\left(u_{i}\right)=b$ for one $u_{i}$ adjacent to $w_{i}, c_{2}(v)=m+1$, and $c_{2}(z)=c_{1}(z)$ otherwise. Then $c_{2}$ is an $(m+1)$-coloring of $G$. Thus, if $\left(^{*}\right)$ holds, $\chi_{d}(G-v) \geq \chi_{d}(G)-1$.

Since $G$ is connected, then $G-v$ is connected (otherwise, there exists an $m$-coloring $c_{1}$ of $G-v$ such that $c_{1}(u)=2$ for all $u$ in $N(v)$ in one 
component and $c_{1}(u)=1$ for all $u$ in $N(v)$ in all other components). Let $l$ be the minimum of all the lengths (number of edges) of shortest nontrivial paths in $G-v$ from vertices in $U$ to vertices in $N(v)$. Thus, $l \geq 2$.

If $l=2$, then there exists a path $u_{i} w_{i} u$ for some $u$ in $N(v)$. Thus, by the double-adjacency condition, $w_{i}$ is adjacent to some vertex $y \neq u_{i}, u$ satisfying $c_{1}(y) \neq 1$. Recoloring $u_{i}$ as $c_{1}(y)$ gives a new $m$-coloring of $G-v$ with not all vertices in $N(v)$ colored the same.

If $l \geq 4$, then $(*)$ is satisfied. So, suppose finally that $l=3$.

If $\left|U^{\prime}\right| \geq 1$, then a shortest path in $G-v$ from $U$ to $U^{\prime}$ begins $u_{i} w_{i} x$ for some $u_{i}$ in $U$ and some $x$ not in $U, U^{\prime}, W$. Thus, $x$ satisfies $\left(^{*}\right)$.

So, suppose $U^{\prime}$ is empty, and suppose, since $d(v) \geq 2$, that $|U| \geq 2$. Then, since $l=3,\left(^{*}\right)$ is not satisfied only if each vertex of $G-v-U$ is adjacent to precisely one vertex of $U$, which we henceforth assume.

If $|U|=2$, then $l=3$ implies that $G-v=P_{3}$. Then $G=C_{5}$, for which $\chi_{d}(G-v)=\chi_{d}(G)-2$.

If $|U| \geq 3$, then if some $d\left(w_{i}\right)=2$, note that since $G-v-u_{i}$ is connected, then $w_{i}$ is adjacent only to $u_{i}$ and to some $w_{j}$ in $W$ with $d\left(w_{j}\right) \geq 3$. Thus, let $c_{2}\left(u_{i}\right)=c_{1}\left(w_{i}\right), c_{2}\left(w_{i}\right)=1, c_{2}(v)=m+1$, and $c_{2}(z)=c_{1}(z)$, otherwise, yielding an $(m+1)$-coloring $c_{2}$ of $G$. If $d\left(w_{i}\right) \geq 3$ for all $w_{i}$ in $W$, then the coloring $c_{2}$ will suffice for any choice of $i$. 


\section{Chapter 9}

\section{Gainer Graphs}

For any graph $G, \chi(G-v) \leq \chi(G)$, since any coloring of $G$ is also a coloring of $G-v$. However, there exist graphs $G$, which we shall call gainer graphs, for which $\chi_{d}(G-v)>\chi_{d}(G)$ holds for at least one vertex $v$ of $G$, called a gainer vertex. For example, consider a graph $G_{1}$ that consists of a 5 -cycle $C=v_{1} v_{2} v_{3} v_{4} v_{5} v_{1}$ and a vertex $v_{6}$ adjacent only to $v_{1}$ and $v_{2}$ (Figure 9.1).

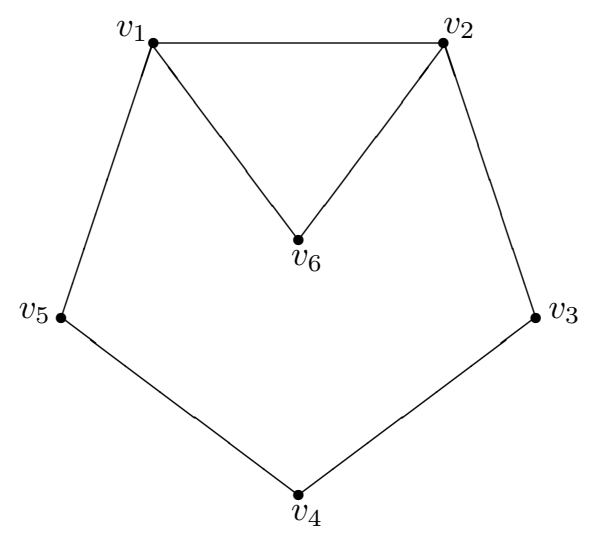

Figure 9.1: $G_{1}$

A dynamic coloring of $G_{1}$ is obtained by coloring $C$ as 1,2,1,3,2,1 and coloring $v_{6}$ as 3 . Thus, $\chi_{d}(G)=3$ and, since $G_{1}-v$ is $C_{5}, \chi_{d}\left(G_{1}-v\right)=5$. 
It has been previously shown that for any graph $G, \chi_{d}(G-v) \geq \chi_{d}(G)-2$, i. e., the removal of a vertex can cause the dynamic chromatic number to decrease by at most two. Thus, we first ask whether, for some positive integer $k$ such as $2, \chi_{d}(G-v) \leq \chi_{d}(G)+k$ holds for all graphs $G$ and all vertices of $G$ or, instead, whether for any given positive integer $k$, there exists a graph $G$ such that $\chi_{d}(G-v) \geq \chi_{d}(G)+k$ for some vertex $v$ of $G$.

The following example shows that no constant upper bound $k$ independent of the graph can be placed on the increase in dynamic chromatic number when a vertex is removed from the graph.

Let $\mathrm{G}$ consist of the bipartite graph $S K_{l}=(X, Y), l=|X| \geq 4$, for which any pair of vertices in $X$ has a unique vertex in $Y$ adjacent precisely to those two vertices (described in Section 4.2), with another vertex $v$ adjacent to all the vertices in $Y$ (which are the vertices of degree two of $S K_{l}$ ). Assign a color of 4 to $v$, a color of 3 to each vertex $x_{i}, 1 \leq i \leq l$, of $X$, and a color of $((i+j) \bmod 2)+1$ to $y$ in $Y$ if $y$ is adjacent to $x_{i}$ and $x_{j}$. Figure 9.2 shows this graph and coloring for $l=4$.

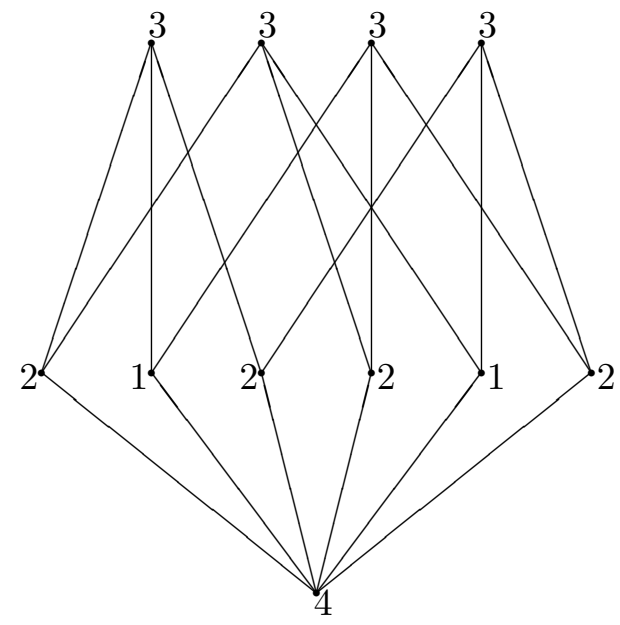

Figure 9.2: $G$ for $l=4$

The coloring is dynamic, so that $\chi_{d}(G) \leq 4$. Since $\chi_{d}(G-v)=\chi_{d}\left(S K_{l}\right)=$ 
$l$, then $\chi_{d}(G-v) \geq \chi_{d}(G)+k$ when $l=k+4$.

Although no constant upper bound independent of the graph may be placed on $\chi_{d}(G-v)-\chi_{d}(G)$ for all graphs, the following theorem shows that an upper bound exists in terms of common graph parameters.

Theorem 20 For any graph $G$ and any vertex $v$ of $G$,

$\chi_{d}(G-v) \leq \chi_{d}(G)+t(v)$,

where $t(v)$ is the number of neighbors of $v$ of degree at least 3. Thus,

$\chi_{d}(G-v) \leq \chi_{d}(G)+d(v) \leq \chi_{d}(G)+\Delta$.

Proof: Any dynamic coloring $c$ of $G$ of $\chi_{d}(G)$ colors will be a dynamic coloring of $G-v$ except that some of the $t(v)$ neighbors $u$ of $v$ having degree at least three in $G$ may not satisfy the double-adjacency condition. To remedy this, use at most $t(v)$ new colors in a coloring of $G-v$ that recolors (in the coloring $c$ induced on $G-v$ ) a neighbor of each $u$ a new color. Since any new color is used only once, then the double-adjacency condition, as well as the adjacency condition, now holds for all vertices in a coloring of $G-v$ of at most $\chi_{d}(G)+t(v)$ colors.

The graph $G$ obtained from $S K_{l}$ does not satisfy with equality the upper bound stated in the theorem since $\chi_{d}(G-v)=\chi_{d}(G)+l-4$ and $t(v)=\left(\begin{array}{l}l \\ 2\end{array}\right)$. The graph $G_{1}$ is an example of a graph that satisfies with equality the upper bound for $t(v)=d(v)=2$.

For each of the two examples of gainer graphs, only one vertex of the graph is a gainer vertex. The following theorem determines whether graphs exist for which every vertex is a gainer vertex.

Theorem 21 A graph exists for which all vertices are gainer vertices. Such graphs do not exist if $\chi_{d}>4$.

Proof: The case for $n \leq 3$ is clear, so let $n \geq 4$ and suppose all vertices of a graph $G$ are gainer vertices. Let $c$ be a dynamic coloring of $G$. Since any $v$ 
is a gainer vertex, then any $v$ is adjacent to some $u$ such that $d(u) \geq 3$ and all $x \neq v$ in $N(u)$ have the same color $c(x) \neq c(v)$. Let any such pair $u, v$ be an arc $(v, u)$ in the partially directed graph $G_{p}$ (denoted $(v, u) \in A\left(G_{p}\right)$ ), which also has $x y \in E\left(G_{p}\right)$ if $x y \in E(G)$ and $(x, y),(y, x) \notin A(G)$, and has $V\left(G_{p}\right)=V(G)$.

Since any $v$ is a gainer vertex, then for any $v,(v, u) \in A\left(G_{p}\right)$ for at least one $u$. Thus, $\sum_{V\left(G_{p}\right)} d^{+}(v) \geq n$.

A vertex $u$ cannot have more than one incoming arc, since if $(v, u)$, $(w, u) \in A\left(G_{p}\right)$, then since $(v, u) \in A\left(G_{p}\right)$ and $w \in N(u)$, then $c(w)=$ $c(x)$ for all $x \neq v$ in $N(u)$, a contradiction of $(w, u) \in A\left(G_{p}\right)$. Thus, $\sum_{V\left(G_{p}\right)} d^{-}(v) \leq n$

Since $\sum_{V\left(G_{p}\right)} d^{-}(v)=\left|A\left(G_{p}\right)\right|=\sum_{V\left(G_{p}\right)} d^{+}(v)$, then $\sum_{V\left(G_{p}\right)} d^{-}(v)=n=$ $\sum_{V\left(G_{p}\right)} d^{+}(v)$. Thus, every vertex has precisely one incoming arc and precisely one outgoing arc, so that the arcs and vertices of $G_{p}$ form one or more vertexdisjoint directed cycles.

Suppose $\chi_{d}(G) \geq 5$. Let $C=v_{1} v_{2} \ldots v_{k} v_{1}$ be one of the vertex-disjoint cycles. The only vertex of $G-v_{i}$ that may not satisfy the double-adjacency condition is $v_{i+1}$ (addition for indices is $\bmod k$ ). Recolor $v_{i+2}$ to be different from $c\left(v_{i+1}\right)$, different from $c(v)$ for any $v \in N\left(v_{i+1}\right)-\left\{v_{i+2}\right\}$, different from $c(v)$ for any $v \in N\left(v_{i+2}\right)-\left\{v_{i+1}\right\}$ and, if $|C| \geq 4$, different from $c(v)$ for any $v \in N\left(v_{i+3}\right)-\left\{v_{i+2}\right\}$. For each of the last three cases, $c(v)$ is the same for all $v$ in the specified set. This coloring is easily seen to be a dynamic coloring of $G-v_{i}$, and since $\chi_{d}(G) \geq 5$, a color does exist to recolor $v_{i+2}$ among the $\chi_{d}(G)$ colors that were used to color $G$. Thus, $\chi_{d}\left(G-v_{i}\right) \leq \chi_{d}(G)$, a contradiction of $v_{i}$ being a gainer vertex of $G$.

Consider the graph $G$ that consists of two vertex-disjoint three-cycles and three additional nonadjacent edges joining pairs of vertices in different cycles. Then $\chi_{d}(G)=3$ and it is easily seen that any vertex of $G$ is a gainer vertex. 


\section{Chapter 10}

\section{A Generalization of Dynamic Coloring}

The concept of dynamic coloring may be generalized to r-dynamic coloring by generalizing the double-adjacency condition in the appropriate fashion.

An r-dynamic coloring of a graph $G$ is thus a map $c$ from $V$ to the set of colors such that

(C1) If $u v \in E(G)$, then $c(u) \neq c(v)$, and

(C2) For each vertex $v \in V(G),|c(N(v))| \geq \min \{r, d(v)\}$

The second condition is called the multiple-adjacency condition.

The minimum $k$ for which $G$ has an $r$-dynamic $k$-coloring is the $\mathrm{r}$-dynamic chromatic number $\chi_{r}(G)$. Thus, $\chi_{d}(G)$, as denoted previously, is identical to what is now denoted as $\chi_{2}(G)$.

From $(\mathrm{C} 1)$ and $(\mathrm{C} 2)$, it immediately follows that $\chi_{r}(G) \geq \min \{r, \Delta\}+1$.

Since any $r$-dynamic coloring of $G$ is a $t$-dynamic coloring of $G$ if $r>t \geq$ 1 , then $\chi_{r}(G) \geq \chi_{t}(G)$ if $r>t \geq 1$ for any graph $G$.

Thus, it is easily deduced that $\chi_{r}\left(K_{n}\right)=n$ for any $r \geq 2$ and that for any particular $n \geq 3$, the chromatic number of $C_{n}$ is the same for all $r \geq 2$.

Theorem 22 If $r \geq 2$, then 


$$
\chi_{r}\left(C_{n}\right)= \begin{cases}5 & \text { if } n=5 \\ 3 & \text { if } n=3 k, k \geq 1 \\ 4 & \text { otherwise }\end{cases}
$$

Proof: For any $r \geq 2, \chi_{r}\left(C_{n}\right) \geq \chi_{2}\left(C_{n}\right)$, and these values for $r=2$ and each $n$ (as stated and proved in Theorem 4) are as given above. Since in each case the proof of Theorem 4 gives a coloring of the cycle having the indicated number of colors and this coloring is an $r$-dynamic coloring for any $r \geq 2$, then the same values are obtained for $\chi_{r}\left(C_{n}\right)$ for any $r \geq 2$.

For the case of trees, a more general result holds than the one previously obtained for $r=2$.

Theorem 23 For any tree, $\chi_{r}(G)=\min \{\Delta, r\}+1$.

Proof: The proof is by induction on $n$.

The result is clearly true for $n=1$ or $n=2$.

Let $G$ be a tree of $n \geq 3$ vertices and let $v$ be a vertex of degree one, adjacent to some vertex $u$. By the induction hypothesis, the tree $G-v$ has a coloring $c$ of $\chi_{r}(G-v)$ colors. A coloring for $G$ is now obtained by choosing an appropriate color for $v$ to extend the coloring.

Suppose first that $\Delta(G) \leq r$. Choose a color for $v$ that is different from $c(u)$ and the at most $\min \left\{d_{G}(u)-1, \Delta(G-v)\right\}$ colors of the other neighbors of $u$. If $d_{G}(u) \leq \Delta(G)=\Delta(G-v)$, this gives a coloring of $G$ of $\chi_{r}(G-v)=\Delta(G-v)+1=\Delta(G)+1$ colors; if $d_{G}(u)=\Delta(G)=\Delta(G-v)+1$, this gives a coloring of $G$ of $\chi_{r}(G-v)+1=\Delta(G-v)+2=\Delta(G)+1$ colors. Thus, a coloring of $\Delta(G)+1$ colors has been obtained, which is minimal and satisfies the theorem, since $\chi_{r}(G) \geq \min \{\mathrm{r}, \Delta(\mathrm{G})\}+1=\Delta(\mathrm{G})+1$ in this case.

Suppose finally that $\Delta(G) \geq r+1$. Then $\Delta(G-v) \geq r$ and so $\chi_{r}(G-v)=$ $r+1$ by the induction hypothesis. Choose a color for $v$ different from $c(u)$ 
and the at most $\min \left\{r, d_{G-v}(u)\right\}-1 \leq r-1$ colors of the other neighbors of $u$ to obtain a coloring of $G$ of $r+1$ colors, which is minimal and satisfies the theorem since $\chi_{r}(G) \geq \min \{r, \Delta(G)\}+1=r+1$ in this case.

Theorem 24 If $k \geq r+1$, then $\chi_{r}\left(K_{i_{1}, \ldots, i_{k}}\right)=k$ if each $i_{j} \geq 1$.

Proof: The unique proper coloring of $K_{i_{1}, \ldots, i_{k}}$ of $k$ colors is also an $r$ dynamic coloring and thus $\chi_{r}\left(K_{i_{1}, \ldots, i_{k}}\right)=\chi\left(K_{i_{1}, \ldots, i_{k}}\right)=k$.

A graph $G$ is defined to be r-normal if $\chi_{r}(G)=\chi(G)$.

Any cycle $C_{n}$, where $n$ is odd and a multiple of three, is $r$-normal for any $r \geq 2$. All other cycles are not $r$-normal for any $r \geq 2$. Any complete graph is $r$-normal for any $r \geq 2$. The only trees that are $r$-normal for at least one value of $r$ are $K_{1}$ and $K_{2}$.

Theorem 25 If any vertex $v$ of a graph $G$ is contained in $K_{k}$ for some $k \geq \min \{r, d(v)\}+1$, then $G$ is r-normal.

Proof: For any proper coloring $c$ of $G,|c(N(v))| \geq \min \{r, d(v)\}$ by the adjacency condition. Thus, $c$ satisfies the multiple-adjacency condition and hence is also an $r$-dynamic coloring. Thus, $\chi_{r}(G)=\chi(G)$ and so $G$ is $r$ normal.

As was shown for $r=2$ in Theorem 14, if $\delta(G)$ is sufficiently large, then $G$ is $r$-normal.

Theorem 26 If $\delta \geq\lfloor(r-1) n / r\rfloor+1$, then $\chi_{r}(G)=\chi(G)$. The lower bound on $\delta$ is the best possible.

Proof: It suffices by Theorem 25 to show that any vertex is contained in a complete subgraph of $r+1$ vertices. Suppose $\delta \geq\lfloor(r-1) n / r\rfloor+1$.

For any vertex $v_{1}$, there exists a vertex $v_{2}$ not in the set $S_{v_{1}}$ of vertices nonadjacent to $v_{1}$, and in general there exists a vertex $v_{t}$ not in the set $\bigcup_{1}^{t-1} S_{v_{i}}$ 
(so that each $v_{i}$ in $\left\{v_{1}, \ldots, v_{t}\right\}$ is adjacent to any other vertex in $\left\{v_{1}, \ldots, v_{t}\right\}$ ) if $\sum_{1}^{t-1}\left|S_{v_{i}}\right|<n$. Since $\left|S_{v_{i}}\right| \leq n-(\lfloor(r-1) n / r\rfloor+1)=\lfloor n / r\rfloor-1$, then $\sum_{1}^{r}\left|S_{v_{i}}\right| \leq r(\lfloor n / r\rfloor-1)<n$.

If $n \geq r+2$, then $G=K_{i_{1}, \ldots, i_{r}}$, where $i_{1}, \ldots, i_{j}=\lfloor n / r\rfloor, i_{j+1}, \ldots, i_{r}=$ $\lceil n / r\rceil$ and $j=\lceil n / r\rceil r-n$, has $\delta(G)=\lfloor(r-1) n / r\rfloor$. Also, $\chi_{r}(G) \geq r+1$ since, otherwise, $\chi_{r}(G)=\chi(G)=r$ and since $G$ is colored uniquely with $r$ color classes, then $|c(N(v))|=r-1<\min \{r, d(v)\}$ for any $v$, a contradiction.

We now consider the problem, treated previously for $r=2$, of what graphs $G$ satisfy $\chi_{r}(G)=n$ if $r \geq 2$.

Theorem 27 For any $r \geq 2, \chi_{r}(G)=n$ if and only if any two nonadjacent vertices of $G$ are adjacent to a vertex of degree at most $r$.

Proof: Suppose $\chi_{r}(G)=n$. If the stated condition is not satisfied for vertices $u$ and $w$, then a coloring $c$ of $G$ of $n-1$ colors in which only $u$ and $w$ are colored the same clearly satisfies the adjacency condition; $c$ also satisfies the multiple-adjacency condition, since for any $v$ not adjacent to both $u$ and $w,|c(N(v))|=|N(v)|=d(v) \geq \min \{r, d(v)\}$, and for any $v$ adjacent to both $u$ and $w, d(v)>r$, and so $|c(N(v))|=|N(v)|-1=d(v)-1 \geq \min \{r, d(v)\}$. Thus, $\chi_{r}(G) \leq n-1$, a contradiction.

Suppose the stated condition holds but $\chi_{r}(G) \leq n-1$. Then some $r$ dynamic coloring $c$ of $G$ of $\chi_{r}(G)$ colors has $c(u)=c(w)$ for two nonadjacent vertices $u$ and $w$. Thus, $u$ and $w$ are adjacent to some vertex $v$ such that $d(v) \leq r$. Hence, $d(v)>|c(N(v))| \geq \min \{r, d(v)\}=d(v)$, a contradiction.

Theorem 27 can be useful for specifying particular graphs satisfying $\chi_{r}(G)=$ $n$ for $r \geq 2$. For example, $P_{3}, C_{4}, C_{5}$, and $K_{n}$ are immediately seen to satisfy the condition of Theorem 2\%. Hence, they each satisfy $\chi_{r}(G)=n$ for $r \geq 2$; this also follows from Theorem 18 and the fact $\chi_{r}(G) \geq \chi_{2}(G)$ if $r \geq 3$. 
Since for all graphs of $n=4$ vertices other than $P_{4}$, any pair of vertices has a common neighbor (of degree at most $\Delta(G) \leq n-1=3$ ), then precisely all five graphs of four vertices other than $P_{4}$ satisfy $\chi_{r}(G)=n$ for $r \geq 3$.

Similarly, for $n=5$ and $r \geq 4$, the only graphs not satisfying $\chi_{r}(G)=$ $n$ are precisely those in which some nonadjacent vertices have no common vertices, i. e., $K_{3}$ with an end of $P_{3}$ adjoined, $C_{4}$ with an end of $P_{2}$ adjoined, $C_{4}+e$ with an end of $P_{2}$ adjoined to a low-degree vertex, and any tree other than $K_{1,4}$.

Theorem 27 allows us to deduce that the only trees satisfying $\chi_{r}(G)=n$ are $K_{1, n-1}$ for $n \leq r+1$, and to deduce that $\chi_{r}\left(K_{n}-e\right)=n$ if and only if $n \leq r+1$. 


\section{Summary}

The number of colors in a minimal dynamic coloring is called the dynamic chromatic number.

One topic of interest was the value of the dynamic chromatic number for important graphs, which was investigated in Chapter 2.

For any of the important theorems involving the chromatic number, a natural question is whether similar theorems exist for the dynamic chromatic number. This was addressed in four different areas by Chapters 3-5. Chapter 3 presented three upper bounds on the dynamic chromatic number that are each quite similar to the corresponding upper bound on chromatic number. Chapter 4 presented results characterizing dynamic vertex-critical graphs for particular values of dynamic chromatic number, and these are similar to results for vertex-critical graphs. Chapter 5 showed that the important theorems for stable graphs for the case of proper colorings do not hold for the case of dynamic colorings.

The difference between the dynamic chromatic number and the chromatic number of graphs is of interest, especially identifying classes of graphs for which the difference is small and identifying whether the difference is bounded for all graphs. Chapter 6 addressed these topics.

The idea of a dynamic coloring is sufficiently different to introduce interesting new topics of graph coloring. Three such new topics were addressed in the next three chapters. Chapter 7 showed that the process of specify- 
ing graphs for which the dynamic chromatic number equals the number of vertices (or one less) is much more complex than for the non-dynamic case. Chapter 8 obtained a characterization of the graphs whose dynamic chromatic number decreases by two with the removal of a vertex. Chapter 9 investigated graphs for which the removal of any vertex causes the dynamic chromatic number to increase.

Chapter 10 introduced a generalization of dynamic coloring. Some of the topics that have been investigated for dynamic coloring in previous chapters were investigated in this chapter for the more general case. 


\section{Recommendations}

Three upper bounds on the dynamic chromatic number were proved in Chapter 3. Additional upper bounds should be investigated, such as an upper bound on the dynamic chromatic number in terms of the number of edges and an upper bound on the sum of the dynamic chromatic number and the dynamic chromatic number of the complement.

The vertex-critical graphs for dynamic chromatic number less than three or equal to the graph order were completely specified in Chapter 4 . Describing the vertex-critical graphs for other dynamic chromatic numbers is recommended.

The difference between the dynamic chromatic number and chromatic number was shown in Chapter 6 to be small for certain regular bipartite graphs, and the case for any regular graph is recommended for investigation.

Gainer graphs were shown in Chapter 9 to exist for dynamic chromatic number equal to three and not to exist for dynamic chromatic number greater than four. The case for dynamic chromatic number equal to four is recommended for investigation.

The $r$-dynamic colorings were introduced and briefly investigated in Chapter 10, and additional investigation is recommended, especially in the areas studied for dynamic colorings. 


\section{Bibliography}

[1] Brooks, R. L., On colouring the nodes of a network, Proc. Cambridge Philos. Soc. 37 (1941) 194-197.

[2] J. A. Bondy and U. S. R. Murty, Graph Theory with Applications, Elsevier North Holland, 1981.

[3] C. Chartrand and L. Lesniak, Graphs and Digraphs, Chapman and Hall, 1996.

[4] Hong-Jian Lai, Bruce Montgomery, and Hoifung Poon, Upper bounds of Dynamic Chromatic Number, accepted by Ars Combinatoria 


\section{Bruce Montgomery}

Bruce Montgomery has received degrees from West Virginia University in 1977 (BS Electrical Engineering) and 1981 (MS Mathematics) and from Carnegie Mellon University in 1984 (MS Electrical Engineering) and 1987 (PhD Electrical Engineering). 